\title{
Morpho-Pathological and Global Transcriptomic Analysis Reveals the Robust Nonhost Resistance Responses in Chickpea Interaction with Alternaria brassicae
}

\author{
Urooj Fatima, ${ }^{1}$ Priyadarshini Bhorali, ${ }^{2}$ and Muthappa Senthil-Kumar ${ }^{1,+}$ \\ ${ }^{1}$ National Institute of Plant Genome Research, Aruna Asaf Ali Marg, P.O. Box No. 10531, New Delhi 110 067, India \\ ${ }^{2}$ Department of Agricultural Biotechnology, Assam Agricultural University, Jorhat-785013, Assam, India \\ Accepted 29 July 2019.
}

\begin{abstract}
Alternaria blight, caused by Alternaria brassicae, causes considerable yield loss in Brassica crops. While several blightresistant varieties have been developed using resistance sources from host germplasm, none of them are entirely successful in imparting durable resistance. This has prompted the exploration of novel gene pools of nonhost plant species. Nonhost resistance (NHR) is a durable form of resistance, comprising pre- and postinvasion layers of defense. We aimed to identify the molecular basis of NHR to A. brassicae and identify the layers of NHR operating in a nonhost, chickpea (Cicer arietinum). To elucidate the layers of NHR operating against $A$. brassicae, we compared the histopathology and infection patterns of $A$. brassicae in C. arietinum and Brassica juncea. Delayed conidial germination, impeded hyphal growth, suppressed appressorium formation, and limited hyphal penetration occurred in the nonhost plant compared with the host plant, implying the involvement of the preinvasion layer of NHR in C. arietinum. Next, we investigated the molecular basis of robust NHR, in $C$. arietinum challenged with $A$. brassicae, by microarray-based global transcriptome profiling. Genes involved in stomatal closure, cuticular wax biosynthesis, cell-wall modification, and secondary metabolite production (contributing to preinvasion NHR) as well as reactive oxygen species (ROS) and cell death (contributing to postinvasion NHR) were found to be upregulated. Consistent with transcriptomic analysis, the morpho-pathological analysis revealed stomatal closure, ROS accumulation, and localized cell death in $C$. arietinum as the defense strategies against $A$. brassicae. Thus, we identified NHR-contributing genes with potential applications in blight resistance gene transfer to $B$. juncea.
\end{abstract}

\section{${ }^{\dagger}$ Corresponding author: M. Senthil-Kumar; skmuthappa@nipgr.ac.in}

Funding: Projects at the M. Senthil-Kumar and P. Bhorali labs are funded by Department of Biotechnology, Ministry of Science and Technology (DBT-NER) twinning project (BT/PR15998/NER/95/145/2015). U. Fatima acknowledges the DBT-SRF fellowship (DBT/2013/NIPGR/68) and National Institute of Plant Genome Research SRF fellowship.

*The $\boldsymbol{e}$-Xtra logo stands for "electronic extra" and indicates that 12 supplementary figures, nine supplementary tables, and a supplementary text file are published online.

The author(s) declare no conflict of interest.

(c) 2019 The American Phytopathological Society
Keywords: Alternaria brassicae, cell death, nonhost resistance, postinvasion defense, preinvasion defense, stomatal defense

Alternaria blight, caused by the fungal pathogen Alternaria brassicae, is one of the most damaging diseases of oilseed brassicas throughout the world (Conn et al. 1990; Kadian and Saharan 1983; Singh Saharan et al. 2016). A. brassicae is a highly infectious and cosmopolitan necrotrophic fungus (Jasalavich et al. 1995; Kadian and Saharan 1983; Tewari 1991). It reduces the yield of B. juncea by up to $47 \%$ in Brassica-growing regions (Meena et al. 2010). A. brassicae infects the host plant at all stages of growth, including the seeds. The sources of primary infection are infected seeds and plant debris. However, under normal conditions, the pathogen spreads through the air and through soil-borne spores, resulting in secondary infection (Thomma 2003). The spores land on the plant surface in the form of conidia, which then develop into germ tubes. The germ tubes grow and penetrate directly or through the stomata into the host epidermal cells by forming appressoria (Cho et al. 2006; Yang et al. 2005). The infection progresses with the appearance of lesions in the form of numerous black sooty spots, leading to severe disease manifestation (Kadian and Saharan 1983; Kolte 1985; Kolte 2002) (Supplementary Fig. S1).

Alternaria blight is currently managed by chemical fungicides. However, in the recent past, efforts have been made to utilize various conventional breeding and modern biotechnological approaches for developing blight-resistant Brassica varieties by exploiting host resistance sources. However, the major bottleneck is the nonavailability of durable resistance sources against $A$. brassicae within the available Brassica crop germplasm. Until this date, no successful and durable strategies have been identified for developing blight-resistant Brassica crops. Hence, there is a need to effectively utilize novel sources of A. brassicae resistance from nonhost plant species.

Nonhost resistance (NHR) is the most durable and effective form of plant immunity against all the isolates of a fungal pathogen (Bettgenhaeuser et al. 2014; Heath 2000; Mysore and Ryu 2004). NHR is expressed by all the variants of a plant species to a given fungal pathogen (Nürnberger and Lipka $2005)$. However, resistance $(R)$ gene-mediated resistance is cultivar-specific and less stable (Gill et al. 2015; Zellerhoff et al. 2010). NHR against fungal pathogens is known to involve multiple layers of resistance, which are mainly divided into preinvasion or postinvasion resistance (Gill et al. 2015; Heath 2000; Zellerhoff et al. 2010). Preinvasion NHR is the layer of resistance wherein the pathogen fails to penetrate the plant, 
while postinvasion NHR operates once the pathogen enters the plant. Reactive oxygen species (ROS) generation and the hypersensitive response (HR) are the main events that occur during postinvasion NHR. These postinvasion responses further check the growth of a fungal pathogen inside the plant (Fonseca and Mysore 2019; Lee et al. 2017). NHR, being a robust and multilayered resistance response, provides a potential option for developing disease-resistant crops.

The transfer of genes involved in NHR against $A$. brassicae from nonhost plants is a promising strategy for developing blight-resistant oilseed Brassica varieties. This warrants the identification of the genes involved in NHR and an understanding of the mechanism by which NHR operates against A. brassicae in nonhost plants. To this end, the prerequisite is to choose potential nonhost plants that can provoke robust and exploitable mechanisms and the underlying genes that are easily transferable to the host plant for imparting durable resistance. Therefore, we first aimed to identify a potential nonhost plant for A. brassicae. Second, we set out to understand the pre- or postinvasion events occurring during the interaction of A. brassicae with the nonhost plant at a morpho-pathological level. At the molecular level, the genes and pathways responsible for NHR against $A$. brassicae are largely unknown. Therefore, we also investigated the transcriptomic changes occurring after pathogen challenge, to identify the genes involved in pre- or postinvasion NHR, by performing microarray and quantitative real-time PCR (RT-qPCR) analysis. In this study, we identified the pre- and postinvasion NHR mechanisms operating in $C$. arietinum against $A$. brassicae.

\section{RESULTS}

\section{Identification of a nonhost plant for A. brassicae.}

We compared the evolutionary distance between nine different plant families belonging to monocots and eudicots affected by A. brassicae (Supplementary Fig. S2). We found that, apart from the Brassicaceae family, which is a host for A. brassicae, several members of the Fabaceae family, including Cyamopsis tetragonoloba (guar), Phaseolus lunatus (lima bean), and Pisum sativum (pea), are also infected by this pathogen (Plantwise Knowledge Bank). Interestingly, however, Cicer arietinum (chickpea), which belongs to the Fabaceae family as well, is not infected by A. brassicae. We explored the intercropping practices of $C$. arietinum with Brassica crops in agricultural fields of India by surveying the literature. Intercropping practices involve the cultivation of two different species together for an extended period (Shekhawat et al. 2012; Singh et al. 2010). We found that, in many parts of India, C. arietinum is intercropped with Brassica spp. and that this cropping pattern is being followed for several decades (Supplementary Table S1). A. brassicae infects Brassica plants but cannot infect $C$. arietinum growing in the same field (Plantwise Knowledge Bank), even though the pathogen gets enough chances to interact with $C$. arietinum during the course of its evolution. Hence, we speculated that NHR mechanisms against $A$. brassicae might be operating in $C$. arietinum and, accordingly, we selected it as the nonhost plant for this study.

\section{Conidial germination, hyphal development, and penetration of $A$. brassicae on nonhost and host plants.}

The infection process of $A$. brassicae on the nonhost (C. arietinum) and host (B. juncea) was compared to investigate the factors responsible for the failure of $A$. brassicae to infect $C$. arietinum plants. The histopathological study to understand the differences in the dynamics of fungal development and the invasion process on $C$. arietinum and $B$. juncea leaves revealed conidial germination on the leaf surface of both species by 12 and $24 \mathrm{~h}$ postinoculation (hpi) (Figs. $1 \mathrm{~A}$ and $\mathrm{K}$ and $2 \mathrm{~A}$ ). Conidial germination on $C$. arietinum was approximately $47 \%$ at $12 \mathrm{hpi}$ and $74 \%$ at $24 \mathrm{hpi}$, compared with $86 \%$ and $99 \%$, respectively, on B. juncea (Fig. 2A). Compared with B. juncea, the frequency of conidial germination was lower, and the process of conidial germination was delayed in $C$. arietinum. At 12 hpi, only one germ tube per conidium emerged in $C$. arietinum, while, in $B$. juncea, more than one germ tube per conidium emerged (Fig. 1A and K, Supplementary Fig. S3). The hyphal length on the leaf surface of $C$. arietinum was significantly lower $(63.22 \mu \mathrm{m})$ than that on $B$. juncea $(230.3 \mu \mathrm{m})$ (Fig. 2C). Lateral branching from aerial hyphae was evident only at 24 hpi in $C$. arietinum compared with $B$. juncea, in which lateral branching of hyphae appeared at 12 hpi (Fig. $1 \mathrm{~B}$ and $\mathrm{L}$ to $\mathrm{N}$ ). In addition, repetitive dichotomous branching of hyphae was not evident in C. arietinum, while, in B. juncea, the hyphal apices showed repetitive dichotomous branching, which formed appressoria at $24 \mathrm{hpi}$ (Fig. 1C and O). The formation of appressoria is the characteristic feature of A. brassicae for its stomatal penetration into the host plant (Giri et al. 2013; Mandal et al. 2018). At 24 hpi in B. juncea, fungal penetration through the stomata was mainly accompanied by the formation of appressoria (Fig. 1D). In C. arietinum, the frequency of germ tubes with appressorium formation was less than $30 \%$, whereas, in B. juncea, more than $70 \%$ of germ tubes formed appressoria at $24 \mathrm{hpi}$ (Fig. 2B). At $24 \mathrm{hpi}$ in $C$. arietinum, aerial hyphae were observed growing over the stomata without any penetration (Fig. 1P). At $24 \mathrm{hpi}$, in C. arietinum, only $2.5 \%$ of fungal penetration attempts through the stomata were recorded, while in B. juncea, more than $60 \%$ fungal penetration through the stomata was observed (Fig. 2B). At $48 \mathrm{hpi}$, the formation of appressoria and penetration attempts through the stomata were evident in C. arietinum (Fig. $1 \mathrm{Q}$ and $\mathrm{R}$ ) and the frequency of stomatal penetration was very low $(10 \%)$ compared with B. juncea $(76 \%)$ (Fig. 2B). In $B$. juncea, direct penetration of fungal hyphae in the junctions between epidermal cells was evident, whereas no such direct penetration was seen in C. arietinum until 48 hpi (Fig. 1E). However, the fungal hyphae led to the formation of swollen appressoria near the epidermal cell junctions in $C$. arietinum (Fig. 1S). In addition, at $48 \mathrm{hpi}$, the aerial hyphal length was significantly lower in $C$. arietinum $(256.88 \mu \mathrm{m})$ than that in B. juncea $(636.02 \mu \mathrm{m})$ (Fig. 2C). In B. juncea, the rapid colonization of fungal hyphae within the intercellular spaces was evident at 48 hpi (Fig. 1F through $\mathrm{H}$ ); however, no such colonization was observed in $C$. arietinum (Fig. 1Q through S). At $72 \mathrm{hpi}$, hyphal differentiation into infection thread-like structures was observed growing on the leaf surface of $C$. arietinum without any extensive hyphal growth or colonization inside the leaf tissue (Fig. 1T). However, at the same timepoint in $B$. juncea, extensive mycelial growth, colonization of fungal hyphae, and reappearance of small hyphae from the stomata were observed (Fig. 1I and J). Moreover, by 7 days postinfection (dpi), no macroscopic disease symptoms were visible in $C$. arietinum, whereas severe necrotic lesions were seen in B. juncea (Fig. 2D).

The microscopic study showed delayed conidial germination, impeded hyphal growth, suppressed appressorium formation, and limited hyphal penetration and colonization of A. brassicae on $C$. arietinum, the nonhost plant. These are the critical stages involved in the infection process of $A$. brassicae. Therefore, the NHR operating in $C$. arietinum appears to actively suppress $A$. brassicae development, penetration, and colonization. These findings imply that the rapid colonization of $A$. brassicae in the host plant occurs within $48 \mathrm{hpi}$, while the infection process is arrested in the nonhost plant. Overall, these 
Infection pattern in the host plant
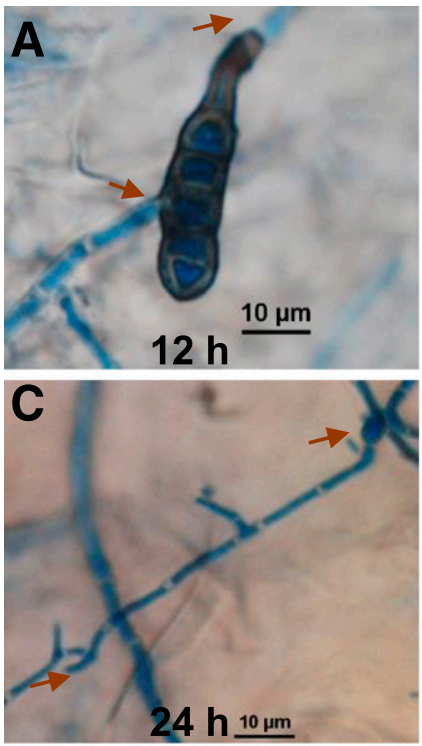

E
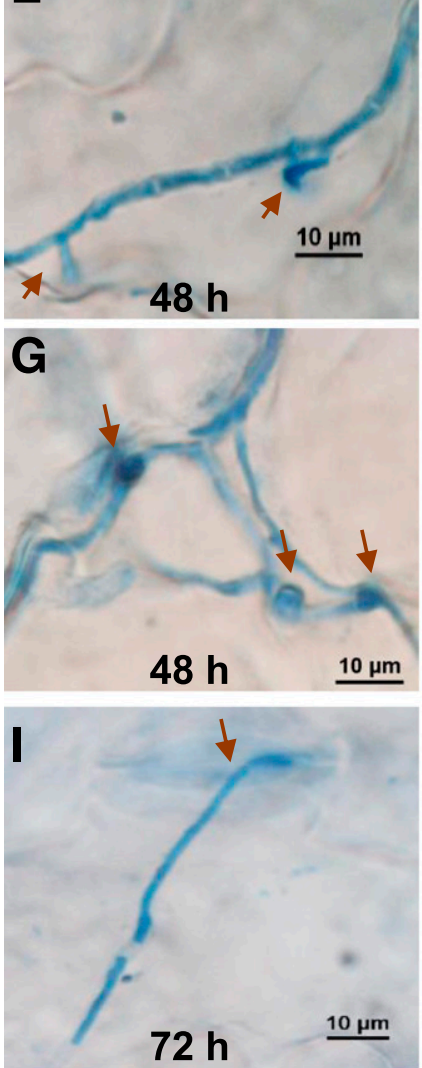
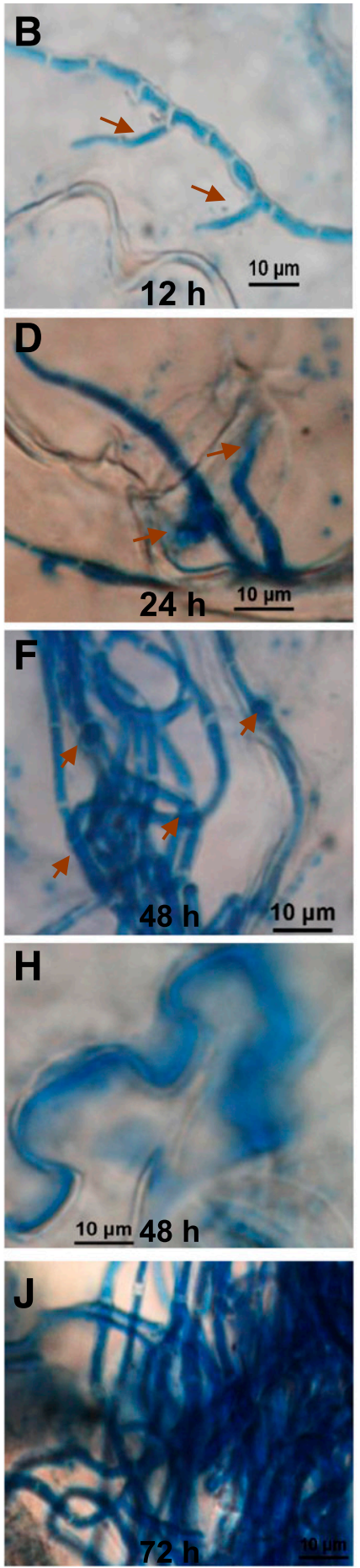

Infection pattern in the nonhost plant
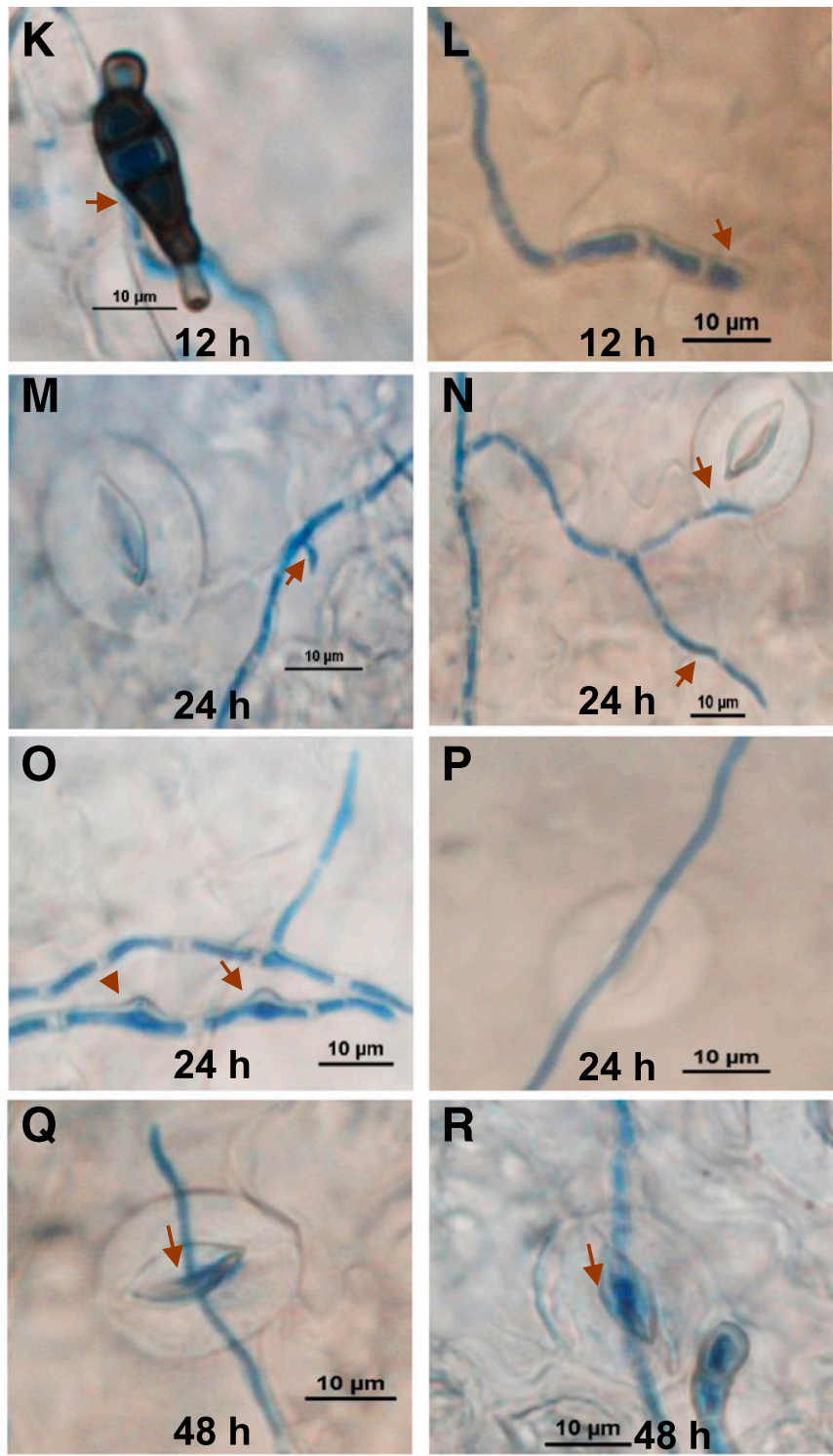

S

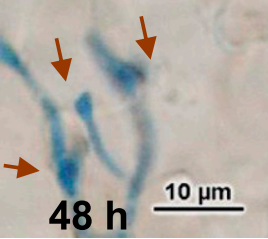

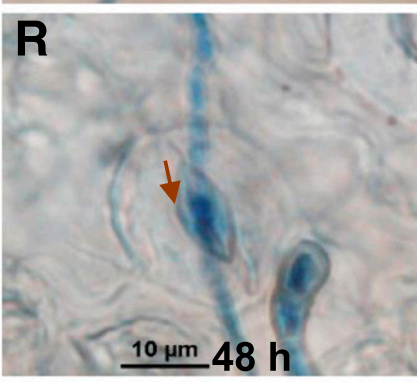

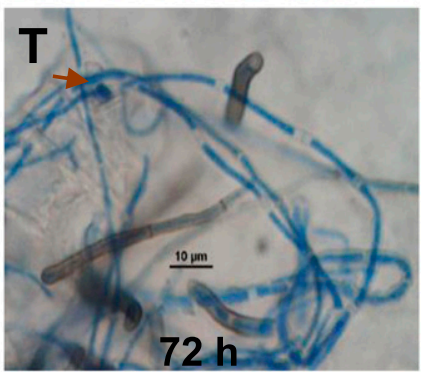

Fig. 1. Comparative microscopic analysis of the infection pattern of Alternaria brassicae on Brassica juncea and Cicer arietinum. Lactophenol aniline blue staining $(0.05 \%)$ of $B$. juncea and $C$. arietinum leaves inoculated with $A$. brassicae was performed, followed by observation using a fluorescent microscope (100× objective). A to $\mathbf{J}$, The infection process of $A$. brassicae in $B$. juncea, the host plant. A, Emergence of hyphae from the intercalary and terminal cells of conidiophores at $12 \mathrm{~h}$ postinoculation (hpi). B, Emergence of lateral hyphal branches from aerial hyphae at $12 \mathrm{hpi}$. C, Repetitive dichotomous branching of the hyphae and appressorium formation at 24 hpi. D, Penetration of the appressorium and hyphal apices into the stomata at 24 hpi. E, Direct penetration of the fungus through the spaces between the epidermal cells at 48 hpi. F, Massive growth and branching of fungal hyphae with multiple appressoria at 48 hpi. $\mathbf{G}$ and $\mathbf{H}$, Proliferation of fungal hyphae with appressoria between the epidermal cell junctions. I, Emergence of mature fungal hyphae through stomatal openings at 72 hpi. J, Extensive hyphal growth at 72 hpi. K to T, The infection process of $A$. brassicae in $C$. arietinum, the nonhost plant. K, Emergence of hyphae only from intercalary cells but not from the terminal cells of conidiophores at $12 \mathrm{hpi}$. L, Growth of hyphae with distended apices on the leaf surface; no lateral hyphal branches are visible at 12 hpi. M, Emergence of small lateral hyphal branches from aerial hyphae at 24 hpi. N, Formation of elongated lateral hyphal branches, passing near the stomata at 24 hpi. O, No repetitive dichotomous branching of hyphae; increased diameter of hyphal cells at 24 hpi. P, Fungal hyphae passing over the stomata without penetrating it at 24 hpi. $\mathbf{Q}$ and $\mathbf{R}$, Stomatal penetration of hyphae accompanied with the formation of appressoria at 48 hpi (as indicated by intense blue staining of the stomatal opening). S, Appressoria were formed from hyphae near the epidermal cell junctions at 48 hpi. T, Hyphal differentiation led to the formation of infection thread-like structures without extensive hyphal growth at 72 hpi. The experiments were repeated twice, and consistent results were seen. The arrows indicate the events described in the respective subsections. 
findings allowed us to identify the suitable timepoints at which the robust NHR response operates in C. arietinum.

\section{Transcriptomic alterations in $C$. arietinum} after $A$. brassicae inoculation.

To elucidate the transcriptomic responses of $C$. arietinum after $A$. brassicae infection, we performed a microarray study. The transcriptomic study was carried out for two timepoints after A. brassicae infection, 24 and 48 hpi. These two timepoints were selected after observing the infection process of $A$. brassicae in $C$. arietinum plants.

Identification of differentially expressed genes (DEGs). The DEGs with $\log _{2}$ fold change $(\mathrm{FC})>1(P<0.05)$ for pathogen-treated samples over the control samples were identified. The putative annotations for the DEGs were extracted from the chickpea transcriptome database (CTDB) (Verma et al. 2015). The unannotated DEGs were not considered in the final list. The complete list of DEGs for both timepoints is provided in Supplementary File S1. The overview of the transcriptomic changes indicating the total number of up- and downregulated DEGs at both timepoints is presented in Figure 3. The data showed the differential expression of 305 transcripts at $24 \mathrm{hpi}$ and 3,498 transcripts at 48 hpi. At 24 hpi, in comparison with mock-treated samples, among 305 DEGs, 91 were upregulated and 214 were downregulated. Similarly, at $48 \mathrm{hpi}, 2,140$ DEGs were upregulated and 1,358 DEGs were downregulated
A

C. arietinum $\square$ B. juncea

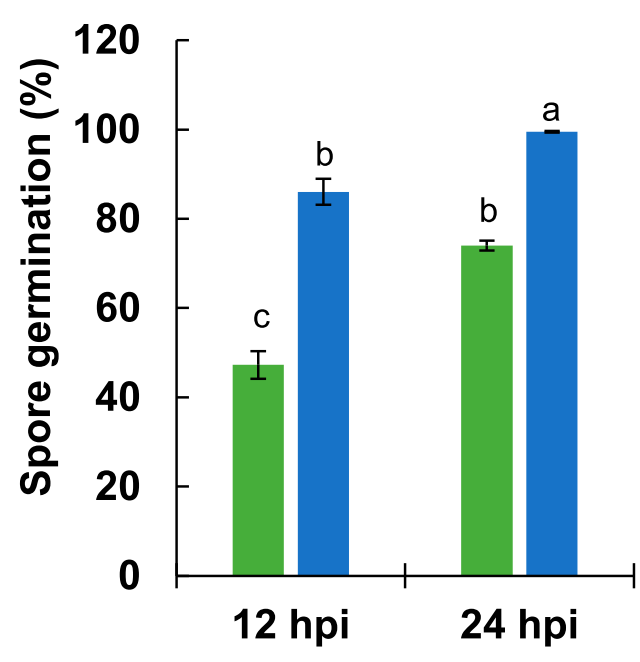

C $\square$ B. juncea

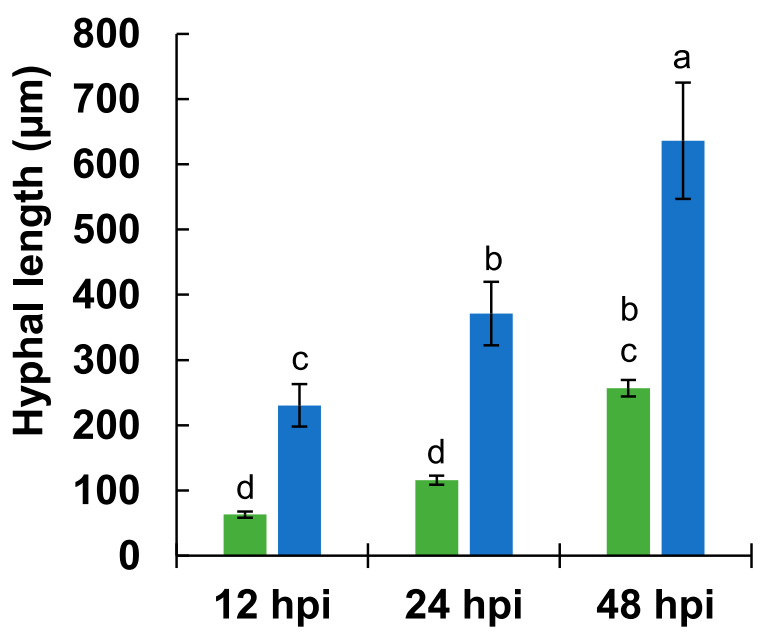

B

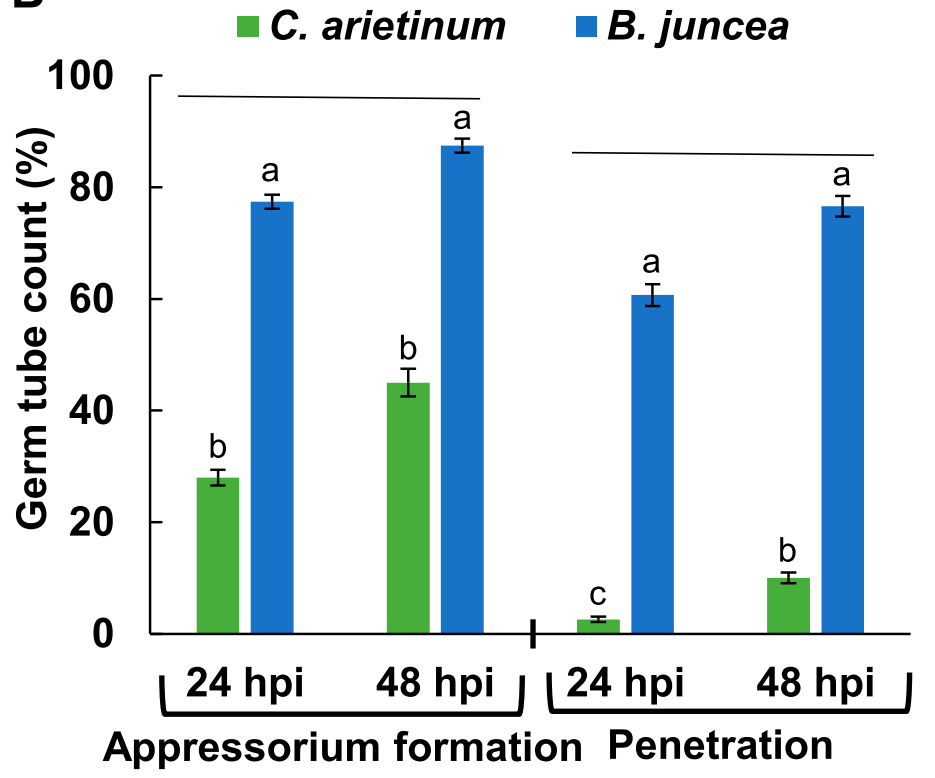

D

B. juncea

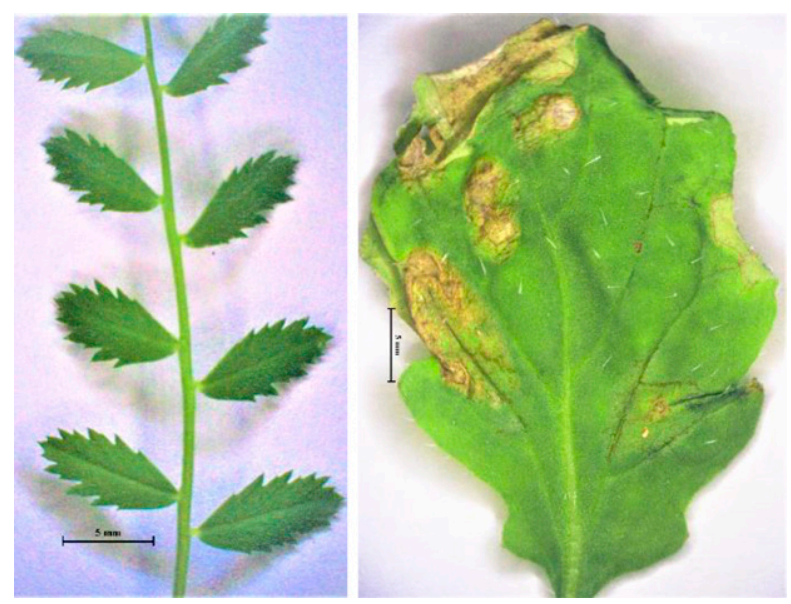

Fig. 2. Cicer arietinum exhibits nonhost resistance against Alternaria brassicae. A, Frequency of spore germination, B, germ tubes with appressorium formation and stomatal penetration, and $\mathbf{C}$, hyphal length elongation were quantified. Lactophenol aniline blue staining $(0.05 \%)$ was performed on leaf samples collected at 12, 24, and $48 \mathrm{~h}$ postinoculation (hpi). Fungal structures were observed in the leaf samples using a light microscope. Spore germination was quantified from nine independent plants, and three leaves from each plant were taken. Appressorium formation and germ-tube penetration were scored from 30 random microscopic fields on 10 independent leaves. Hyphal length was measured as an average of 50 random spores from seven independent leaves. Experiments were repeated at least twice, and consistent results were observed. One-way analysis of variance with Tukey's correction was applied; significant differences $(P<0.05)$ are indicated by different letters. D, The phenotypes of $C$. arietinum and Brassica juncea leaves inoculated with $A$. brassicae at a spore concentration of $5 \times 10^{4}$ spores $/ \mathrm{ml}$. The inoculated plants were kept in $100 \%$ humidity for 3 days after inoculation, after which the humidity was reduced to $70 \%$ till the end of the experiment. The photographs were taken at 7 days postinoculation under a $0.5 \times$ objective of a Stereozoom AZ100 microscope. 
compared with the mock control (Fig. 3A). Furthermore, a comparison of both timepoints revealed that 63 and 2,112 DEGs were uniquely upregulated at 24 and $48 \mathrm{hpi}$, respectively, while 109 and 1,253 DEGs were uniquely downregulated at 24 and $48 \mathrm{hpi}$, respectively. Among the common genes, only 28 DEGs were upregulated and 105 were downregulated for

A

Downregulated genes - Upregulated genes

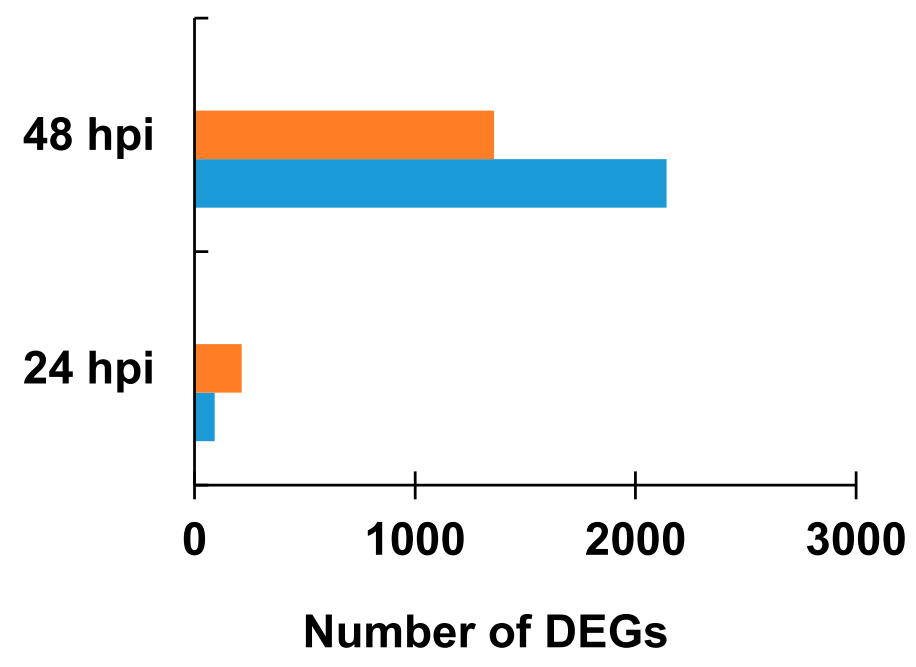

both timepoints (Fig. 3B). As indicated by the presence of a considerably larger number of transcripts at $48 \mathrm{hpi}$, the modulation of the transcriptome response after $A$. brassicae treatment seems to be more robust at 48 than at $24 \mathrm{hpi}$.

The expression profiles of the top 20 upregulated and top fivedownregulated genes after pathogen infection at 24 and

\section{B}

\section{Upregulated genes}
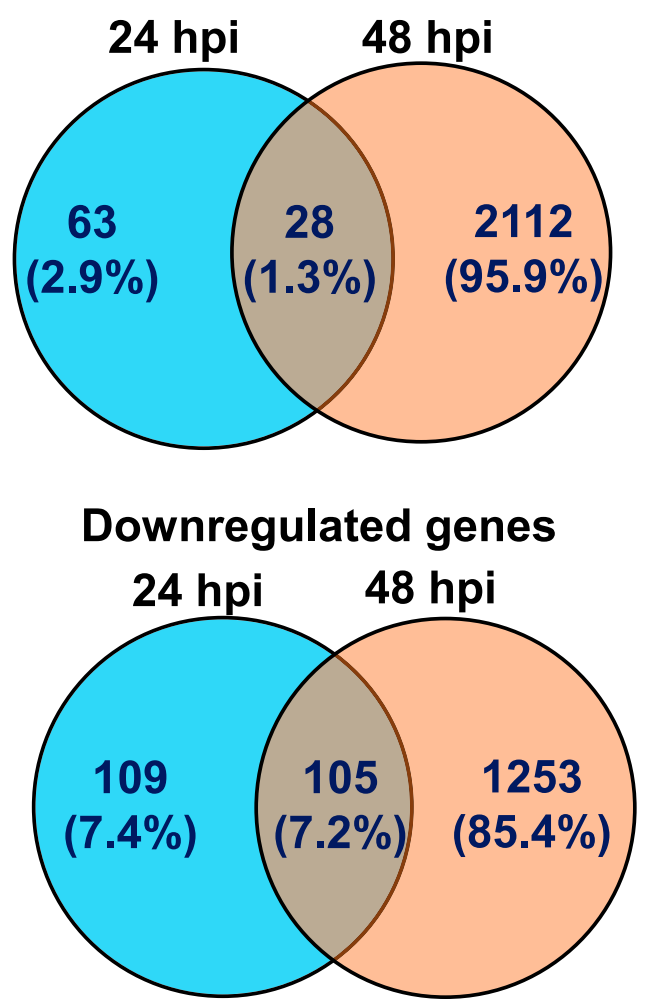

\section{C \\ 24 hpi

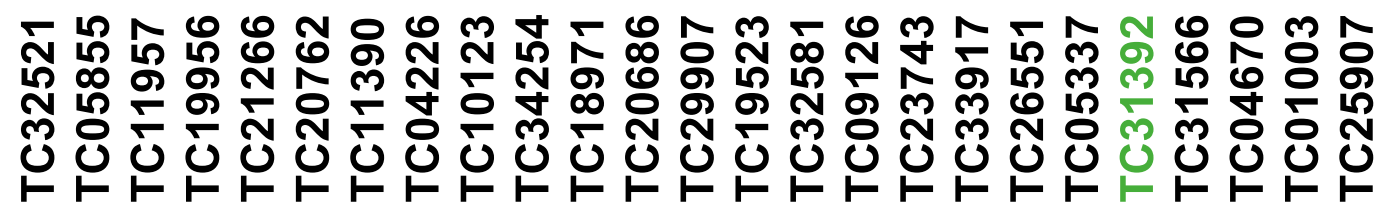

D

48 hpi

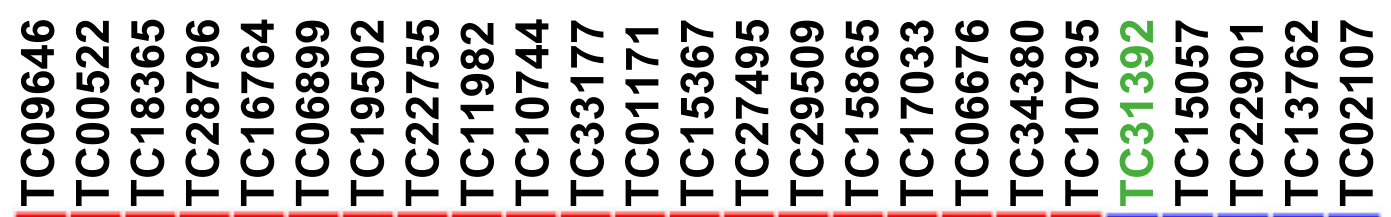

Fig. 3. Overview of differentially expressed genes in Cicer arietinum upon inoculation with Alternaria brassicae. A, Number of up- and downregulated differentially expressed genes (DEGs) at 24 and $48 \mathrm{~h}$ postinoculation (hpi). B, Venn diagrams show the number of common and unique genes up- and downregulated at 24 and 48 hpi. C and D, Heat maps show the expression level of the top 20 upregulated and top five downregulated genes at 24 and 48 hpi. The alteration in the transcriptome was studied in C. arietinum inoculated with A. brassicae at 24 and 48 hpi. The DEGs were obtained at 24 and 48 hpi over the mock control. For A through D, DEGs with a minimum fold change (FC) cut-off $=1$ ( $\log _{2}$-transformed values) and $P<0.05$ were considered for analysis. Heat maps were generated, through Morpheus software, using the FC values. The scale bar indicates the range of FC. Genes commonly modulated at 24 and 48 hpi are in a different shade in the text. 
$48 \mathrm{~h}$ are shown in Figure 3C and D and Supplementary Table S2. At 24 hpi, most of the up- and downregulated DEGs were under the category of genes encoding enzymes such as protein disulfide isomerase-like protein, nucleoside triphosphate hydrolases, cytosine methyltransferase MET1, peptidase S41, protein tyrosine phosphatase-like, carboxyl-terminal domain phosphatase-like 2, and haloacid dehalogenase-like hydrolase. This indicates the reprogramming of genes related to catalytic activities, phosphatase activities, and other protein modifications at $24 \mathrm{hpi}$. At $48 \mathrm{hpi}$, the gene encoding the sugar transporter nodulin MtN3 family protein (orthologous to AtSWEET7 in Arabidopsis) was highly expressed in C. arietinum. Other members of the SWEET family of sugar transporters in Arabidopsis, rice, and grapevine have been associated with plant defense and pathogenesis (Chen 2014; Chen et al. 2010, 2012). Among the top upregulated genes, most were related to the defense response, e.g., genes encoding cysteinerich receptor-like protein, RNI-like super family protein DREB2A-interacting protein 2, and senescence-associated gene 101. Our results suggest that the $C$. arietinum transcriptome at 24 hpi is significantly different from that at 48 hpi.

To validate the results from microarray data, RT-qPCR analysis was performed for the 11 genes from the list of upregulated genes at both the timepoints. The relative expression levels of these genes obtained from RT-qPCR are represented in Figure 4. The RT-qPCR analysis depicting the expression pattern of these genes authenticated the microarray data.

Functional annotation of DEGs. We also categorized the DEGs obtained at 24 and 48 hpi on the basis of gene ontology (GO), using the Arabidopsis orthologs for C. arietinum DEGs. The DEGs at 24 and 48 hpi were categorized into biological process, molecular function, and cellular component, using the GO tool from The Arabidopsis Information Resource (TAIR). We found overlap in the enrichment of up- and downregulated DEGs (for both the timepoints) in GO biological processes such as metabolic and cellular processes, in GO molecular function, such as enzymatic activity, protein-binding activity, and transferase activity, and GO cellular components, such as nucleus, cytoplasm, and the intracellular component (Fig. 5; Supplementary Fig. S4). Besides the TAIR GO annotation database, we used agriGO as a tool to further subcategorize the different biological processes. At $48 \mathrm{hpi}$, the $C$. arietinum transcriptome exhibited the upregulated DEGs explicitly related to subcategories such as response to biotic stress, immune response, and programmed cell death (PCD) (Fig. 5); however, these categories were not found to be enriched at 24 hpi (Supplementary Table S3). These results indicate that the heightened defense response is activated at $48 \mathrm{hpi}$.

\section{Differential expression of defense- and phytohormone-related genes.}

MapMan was used, as an additional microarray data analysis tool, to elucidate the genes and pathways involved in the defense response against $A$. brassicae. The transcriptomic data in terms of the number of upregulated genes related to the defense response against the pathogen differed considerably between 24 and 48 hpi (Supplementary Fig. S5). At 24 hpi, only a few genes related to defense processes, which are mainly involved in pathogen detection, protein degradation via ubiquitination, and RNA-mediated silencing, were found to be upregulated (Fig. 6A; Supplementary Table S4). Intriguingly, some defenserelated genes were also found to be downregulated (Fig. 6A). In addition, at $48 \mathrm{hpi}$, the transcriptomic response was majorly altered for the genes encoding proteins involved in signaling, protein degradation via ubiquitination, pathogenesis-related protein, heat-shock protein, cell-wall modification, and secondary metabolism. Most of the genes related to biotic stress pathways were found to be upregulated at $48 \mathrm{hpi}$, including those encoding proteins functioning as immune receptors (Fig. $6 \mathrm{~B})$. The expression of genes encoding different receptor-like kinases (RLKs), which play a crucial role in the perception of signals from a pathogen and activation of defense response, was also upregulated. Likewise, the expression of genes involved in the ubiquitination-mediated defense response, systemic acquired resistance-mediated disease resistance and NHR were found to be upregulated (Fig. 6B).

Further, the transcriptome analysis revealed that the genes involved in hormone biosynthesis, transport, and signaling were upregulated at 48 hpi after A. brassicae infection (Supplementary Fig. S6). We observed the upregulation of various genes associated with abscisic acid (ABA) biosynthesis, ABA transport, and the ABA-mediated signaling pathway (Supplementary Table S5), indicating involvement of ABA in the plant defense response against $A$. brassicae infection. In addition, we also observed the differential expression of genes associated with other hormones, such as cytokinin, ethylene, jasmonic acid, and others. Overall, these results indicate that the interaction of $A$. brassicae with $C$. arietinum impacts the expression of genes underlying the hormones related to defense and growth processes.

\section{Identification of the genes involved in the pre- and postinvasion layers of NHR.}

We identified a few DEGs from the transcriptomic data that might be involved in the preinvasion layer of NHR, as assessed from the literature (Supplementary Fig. S7). A. brassicae penetrates directly by digesting the cuticle or through the stomata into host epidermal cells. We observed that genes involved in stomatal closure and stomatal opening were up- and downregulated, respectively, at both timepoints (Supplementary Table S6). At 24 hpi, open stomata 1 (OST1), which encodes the calcium-independent ABA kinase and is involved in stomatal closure (Kim et al. 2018; Mustilli et al. 2002), was upregulated. At $48 \mathrm{hpi}$, the gene encoding protein phosphatase 2ca (PP2CA), involved in stomatal closure, was upregulated. $F A M A$, the gene involved in stomatal development, thereby helping in plant defense against fungal pathogens ( $\mathrm{Li}$ et al. 2018), was also upregulated. Besides this, the expression of genes involved in cuticle development and wax biosynthesis, which might be involved in preinvasion NHR, was upregulated as well. Taken together, these findings support that stomatal closure and cuticle thickening might be preinvasion NHR strategies that prevent $A$. brassicae entry inside $C$. arietinum tissues.

Additionally, secondary metabolites play an important role as chemical barriers during pre- or postinvasion defense against a pathogen. The DEGs were mapped onto different pathways involved in secondary metabolism (Supplementary Fig. S8). At 48 hpi, genes related to the production of secondary metabolites, i.e., flavonoids, phenylpropanoids, alkaloids, carotenoids, and lignins, were found to be upregulated. Further, the cell wall is the primary physical barrier preventing the pathogen from entering inside the plant cell. DEGs involved in cell-wall modifications such as cell-wall thickening and cell-wall lignification were also found to be upregulated at $48 \mathrm{hpi}$. The expression of genes encoding cinnamyl alcohol dehydrogenase 5 (the key enzyme involved in lignin biosynthesis and cell-wall lignification) (Tronchet et al. 2010) and callose synthase 3 (involved in callose deposition) (Ellinger and Voigt 2014) was validated by RT-qPCR (Supplementary Fig. S9). Thus, during pre- or postinvasion defense, $C$. arietinum might be inducing the expression of genes related to secondary metabolite production and cell-wall modification. 

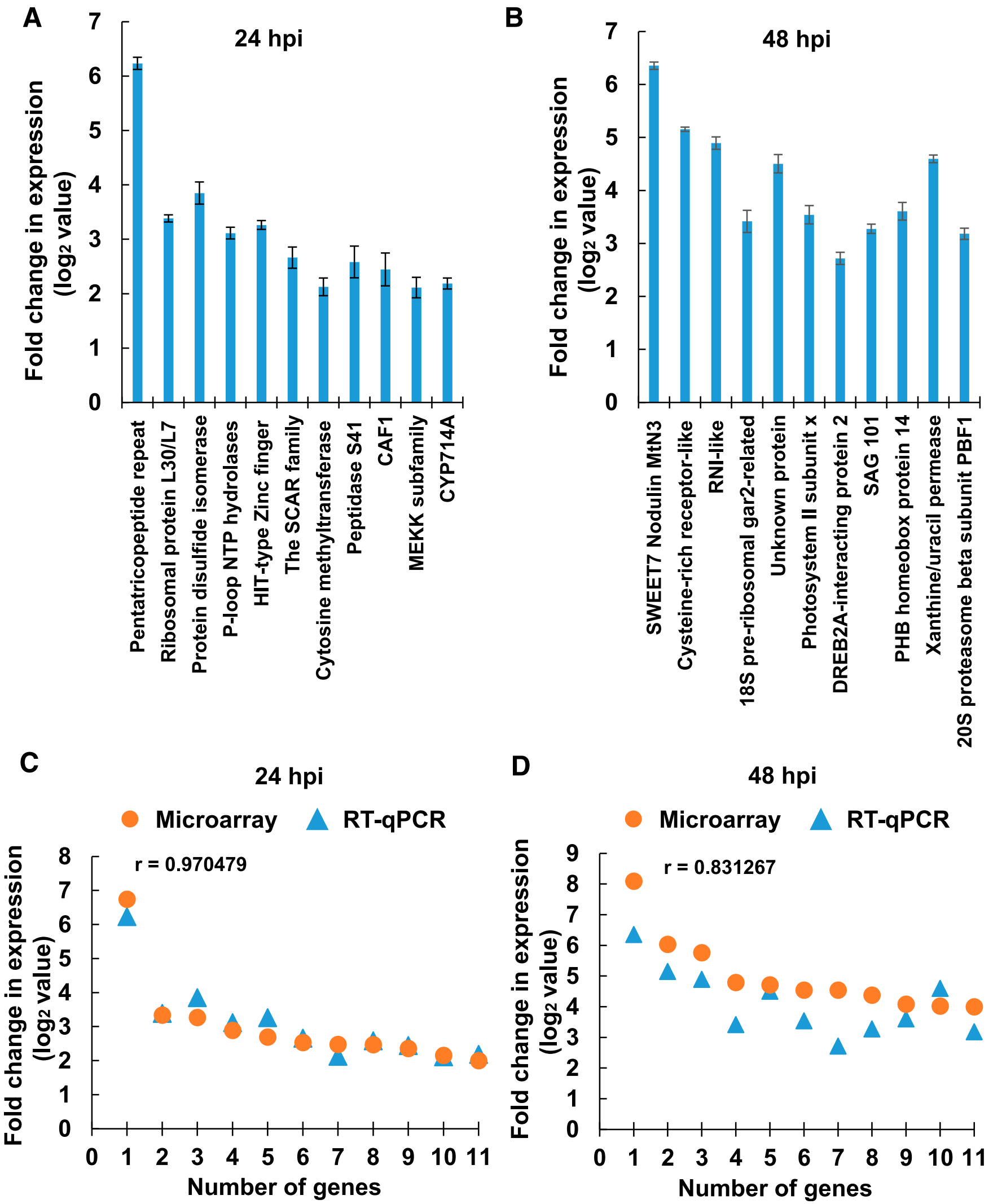

Fig. 4. Validation of microarray data by quantitative real-time PCR (RT-qPCR) analysis. A and B, Transcript expression of the 11 selected genes from the microarray data were quantified by RT-qPCR in Cicer arietinum samples harvested at 24 and $48 \mathrm{~h}$ postinoculation (hpi) after Alternaria brassicae inoculation. The expression values were normalized against CaACTIN2 as a reference gene. The graphs indicate the $\log _{2}$ fold change (FC) values for all the genes, which were calculated over the mock control. The data were obtained as the mean of three biological replicates. Error bars indicate the standard error of the mean. Student's $t$ test at $P<0.05$ was applied. Significant differences were observed for all the genes compared with the mock control. The experiments were repeated twice, and consistent results were seen. C and D, Comparison of FC values $\left(\log _{2}\right)$ from microarray and RT-qPCR data for selected genes, at 24 and $48 \mathrm{hpi}$, using scatter plot analysis. The letter ' $r$ ' represents the correlation coefficient, which was calculated from the data obtained from the microarray and RT-qPCR. The critical value (c) is 0.602 when the degree of freedom $(d f=n-2)$ is 9 , where $n$ represents the total number of genes. $\mathrm{r}>\mathrm{c}$ at both the timepoints indicated significant correlation $(P<0.01)$ between the dataset obtained from microarray and RT-qPCR analyses. 
Postinvasion NHR directs HR-mediated cell death to further limit fungal pathogen growth. For example, we identified a couple of upregulated DEGs at $48 \mathrm{hpi}$, SAG1O1 and $\mathrm{NHOl}$ (Fig. $6 \mathrm{~B})$, that might contribute to postinvasion defense through the manifestation of HR-mediated cell death ( $\mathrm{Lu}$ et al. 2001; Maeda et al. 2010; Makandar et al. 2015). Moreover, the accumulation of ROS, leading to cell death and HR, is the common manifestation of NHR used by the plant to limit nonhost pathogen growth (Greenberg and Yao 2004). ROS are signaling molecules that activate the defense response against a nonhost pathogen (Miller et al. 2010; Rojas et al. 2012). HR restricts pathogen growth by triggering rapid and localized cell death at the infection site (Oh et al. 2006). At 24 hpi, we observed the upregulation of the cupredoxin superfamily protein, which is involved in the oxidation-reduction process and ROS generation. Further, the enrichment of biological processes related to PCD and HR was found at 48 hpi (Supplementary Fig. S10). Some of the prominent upregulated genes were radical-induced cell death1 (RCD1), downy mildew resistant 6 (DMR6; encoding a putative 2OG-Fe(II) oxygenase involved in the ROS-mediated defense response), and flavin-dependent monooxygenase 1 (FMO1; involved in PCD at the infection site) (Supplementary Table S8). These results highlight that the postinvasion defense response of $C$. arietinum involves ROS generation, HR, and cell death at the infection site to prevent the growth of A. brassicae.

\section{Experimental validation of the processes related to pre- and postinvasion NHR in $C$. arietinum.}

Transcriptome analysis revealed the upregulation of genes involved in stomatal closure, in C. arietinum upon inoculation with $A$. brassicae, at 24 and $48 \mathrm{hpi}$. We therefore quantified the expression of $O S T 1$ (at $24 \mathrm{hpi}$ ), PP2CA, and FAMA (at $48 \mathrm{hpi}$ ) genes in $C$. arietinum after inoculation with $A$. brassicae by RT-qPCR. The expression of these genes was upregulated to a similar level as that observed in the microarray study (Fig. 7A, B, and C). Moreover, to investigate whether stomatal closure is one of the NHR strategies in $C$. arietinum for combating $A$. brassicae, we observed the intensity of stomatal closure in mock-treated and A. brassicae-treated C. arietinum plants at 24 and 48 hpi. For this, the stomatal aperture index (SAI) was calculated by measuring the width and length of the stomata by light microscopy. The SAI values for $A$. brassicae-treated plants were significantly lower than those of mock-treated plants at both 24 and 48 hpi (Fig. 7D), implying substantial stomatal closure in $C$. arietinum after A. brassicae inoculation. This indicates that the preinvasion NHR in $C$. arietinum may lead to stomatal closure in order to prevent penetration of $A$. brassicae.

Our microarray study also revealed the upregulation of genes related to ROS-induced cell death in $C$. arietinum upon inoculation with $A$. brassicae. We further validated the expression of a few genes related to ROS generation and defenseinduced cell death by RT-qPCR analysis (Supplementary Fig. S11). Next, we confirmed the presence of ROS-associated cell death, a postinvasion NHR mechanism, at the infection site in C. arietinum. The accumulation of ROS, assessed by staining mock-treated and A. brassicae-treated $C$. arietinum leaves at 24,48 , and 72 hpi with 3,3' -diaminobenzidine (DAB), revealed very low $\mathrm{H}_{2} \mathrm{O}_{2}$ accumulation at $24 \mathrm{hpi}$, which slowly increased at 48 and 72 hpi in A. brassicae-treated plants compared with the mock-treated ones. However, we did not observe any macroscopic cell death or visible HR symptoms in $C$. arietinum after A. brassicae inoculation (Fig. 2D). The extent of microscopic cell death in $C$. arietinum was assessed by trypan blue staining and localized cell death, in the form of small blue spots, was observed on the leaf. Cell death was much less at 24 hpi compared with 48 hpi and a slight increase was observed by 72 hpi (Fig. 7E). Interestingly, the pattern of ROS accumulation consistently corresponded to cell death. These results suggest that postinvasion NHR in $C$. arietinum leads to ROS accumulation and highly localized microscopic cell death to restrict the growth of $A$. brassicae.

\section{DISCUSSION}

NHR is considered an effective and powerful source of durable resistance (Gill et al. 2015; Heath 2000; Zellerhoff et al.

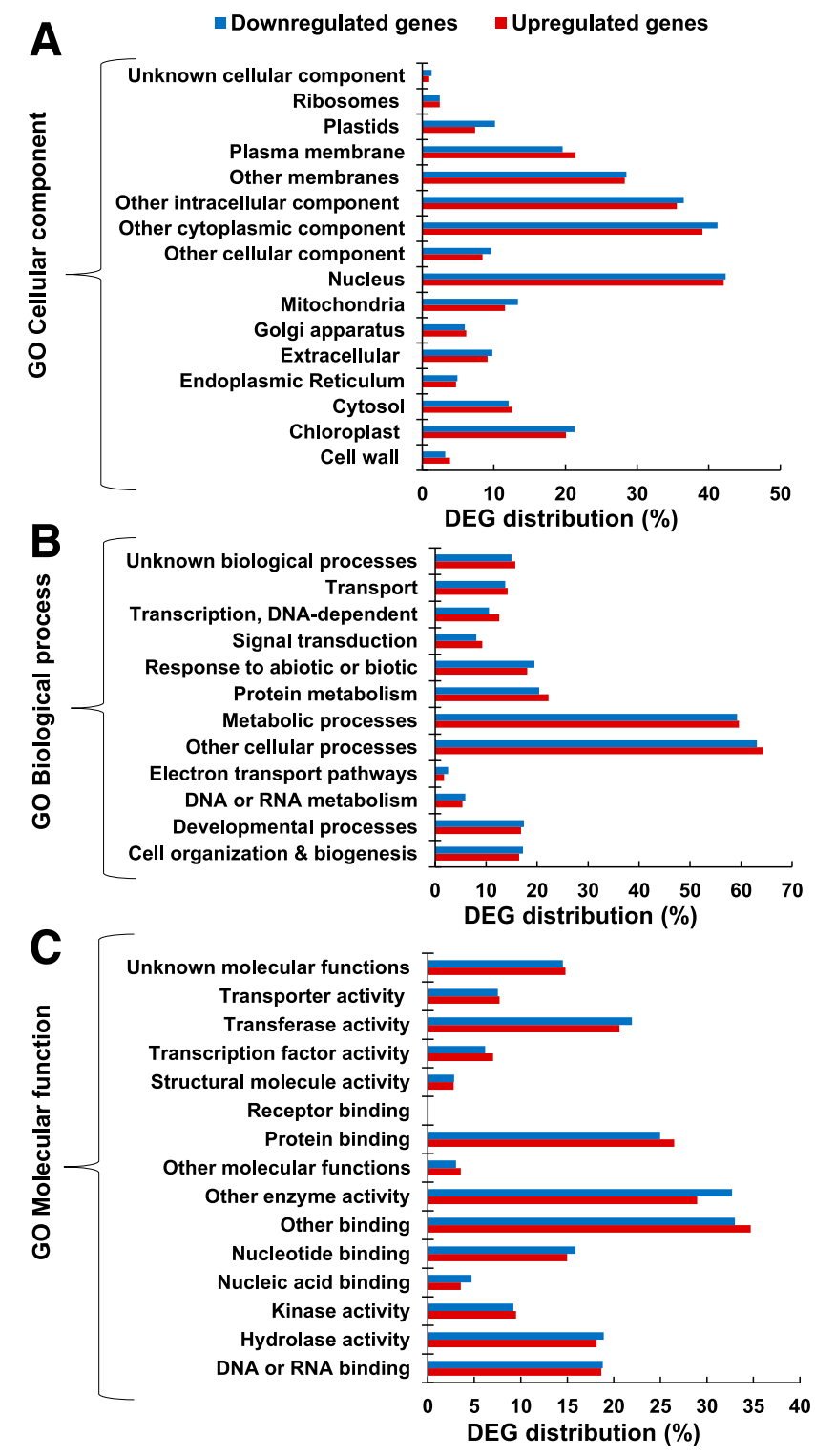

Fig. 5. Gene ontology enrichment analysis for functional categorization of genes in Cicer arietinum upon inoculation with Alternaria brassicae. A to C, Enrichment of gene ontology (GO) terms for cellular component, biological process, and molecular function in $C$. arietinum at $48 \mathrm{~h}$ postinoculation. For GO enrichment, Arabidopsis orthologs of differentially expressed $C$. arietinum genes $\left(\log _{2}\right.$ fold change $\left.>1, P<0.05\right)$ were obtained from the Chickpea Transcriptome Database. The genes were classified among various $\mathrm{GO}$ categories, and GO enrichment was performed using the TAIR GO tool. The bar diagram represents the enriched broad GO terms. In each pair of bars, the lower one indicates the percentage distribution of upregulated genes, and upper one indicates the percentage distribution of downregulated genes under the respective GO terms. More precise functional categorization of the transcripts falling under different subcategories was performed by agriGO singular enrichment analysis with default settings. 

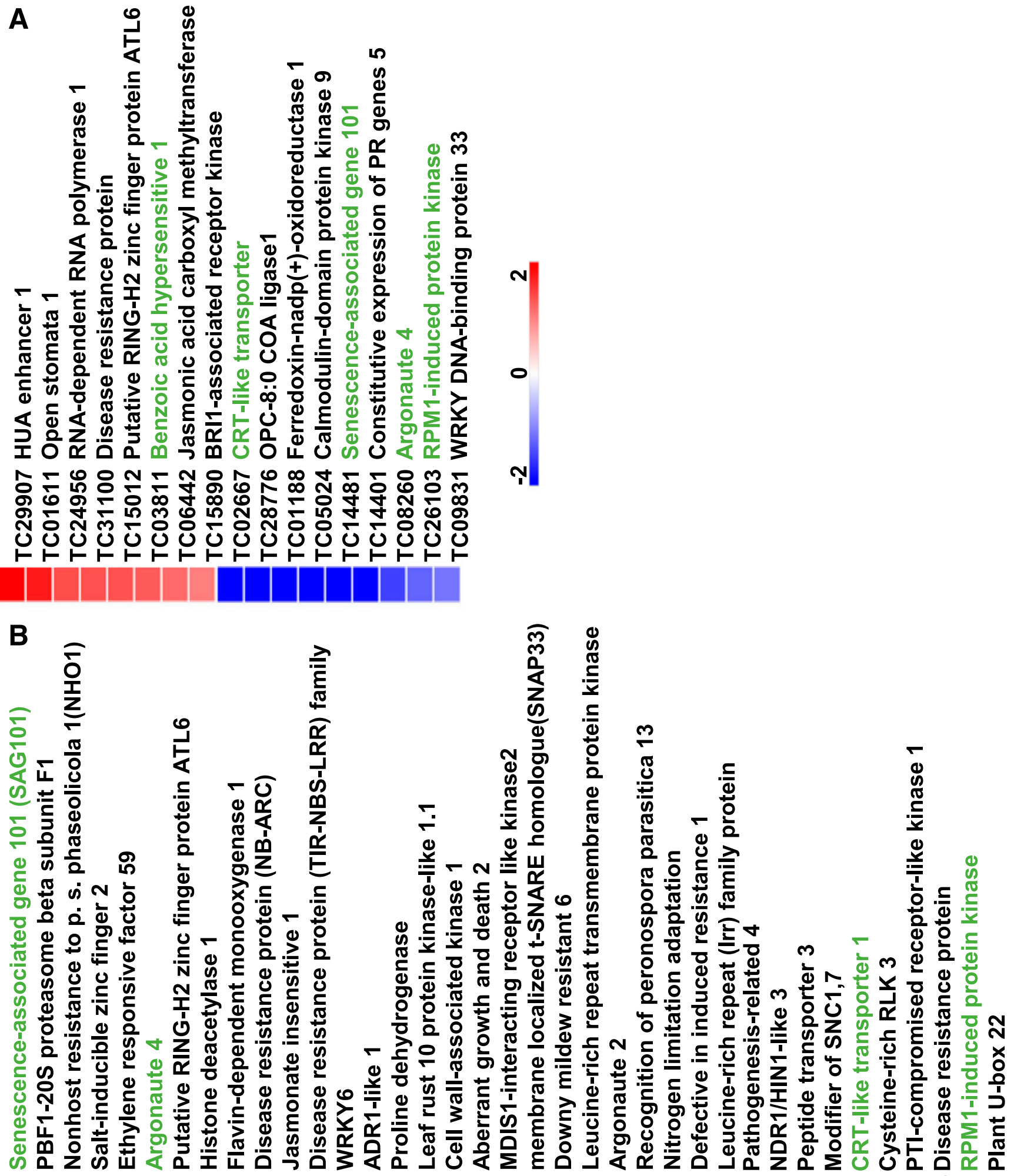

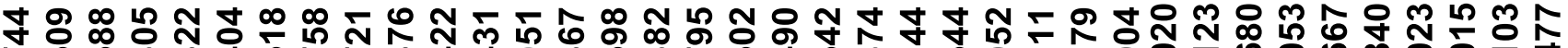

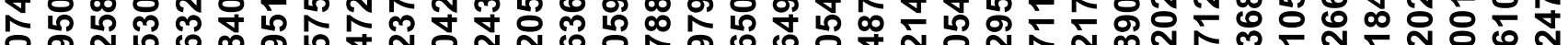
\begin{tabular}{l}
0 \\
\hline
\end{tabular}

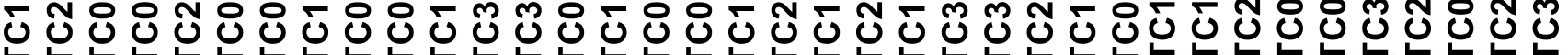

Fig. 6. Expression analysis of defense-related transcripts in Cicer arietinum after inoculation with Alternaria brassicae. A and B, Expression level of genes involved in defense after $A$. brassicae inoculation, at 24 and $48 \mathrm{~h}$ postinoculation (hpi). The differentially expressed genes (DEGs) were obtained at 24 and 48 hpi compared with the mock control. The DEGs with a minimum fold change (FC) cut-off of 1 ( $\log _{2}$-transformed values) and $P<0.05$ were considered for the analysis. The list of genes was curated manually and by using agriGO. Heat maps were generated through Morpheus software, using the FC values. Genes commonly modulated at 24 and 48 hpi are in a different shade in the text. 
2010). Owing to its robust nature, NHR can be utilized as a resistance source to develop crops with durable resistance to necrotrophic fungal pathogens. Various studies have reported on the host-necrotrophic pathogen interaction involving A. brassicae and B. juncea (Giri et al. 2013; Mandal et al. 2018; Singh Saharan et al. 2016). Here, we successfully identified the suitable timepoints at which the robust NHR response operates in the nonhost $C$. arietinum, and, for the first time, we illustrated the interaction of $A$. brassicae with this nonhost. Using morpho-pathological analysis together with transcriptomics, we identified NHR-contributing genes and the NHR layers operating in the resistance of $C$. arietinum to A. brassicae. The transcriptome study of $C$. arietinum against $A$. brassicae reflects the robust changes in the defense response at later stages
A CaOST 1

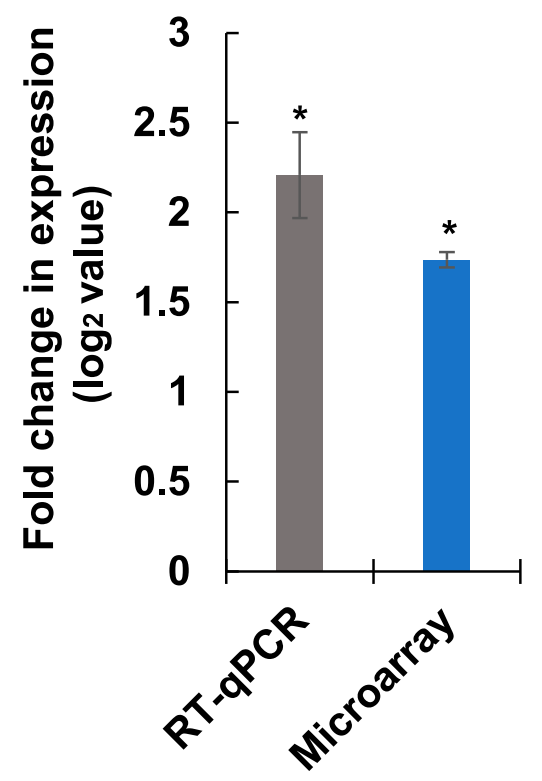

D

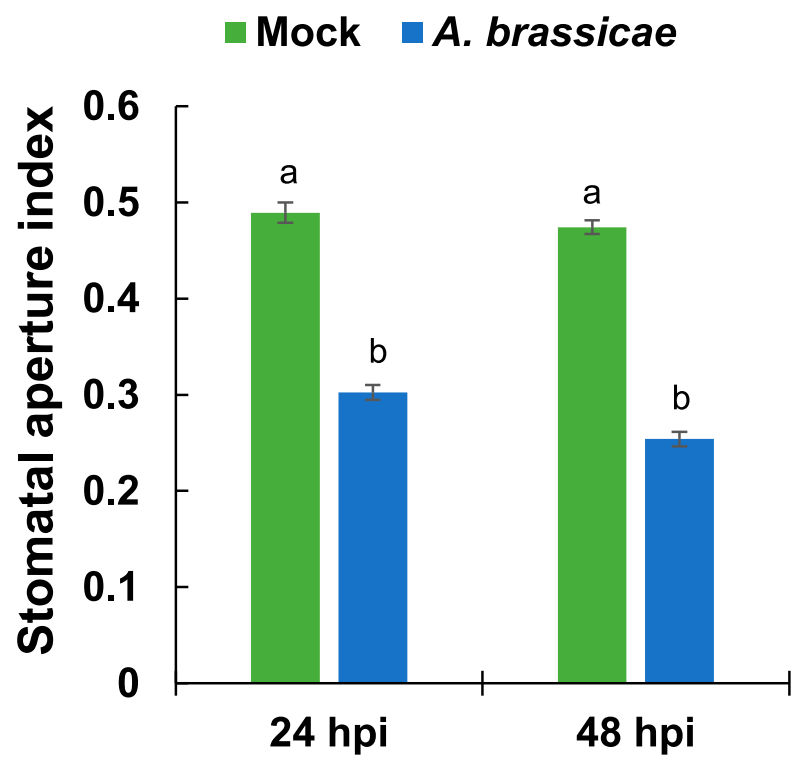

B

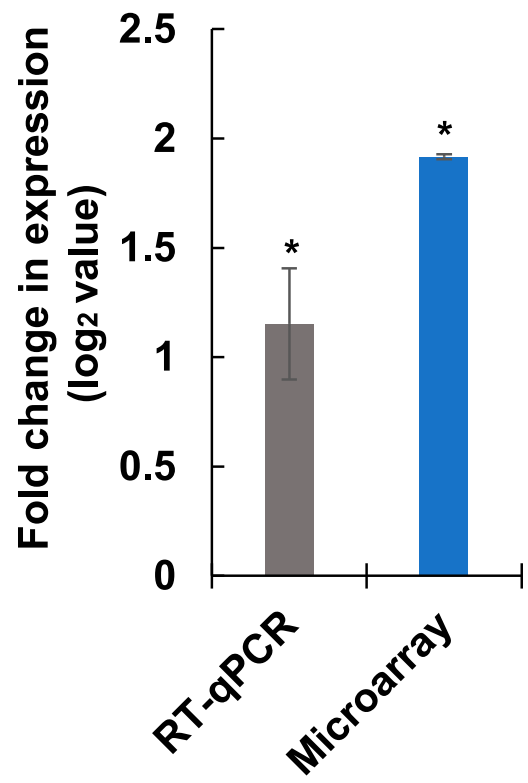

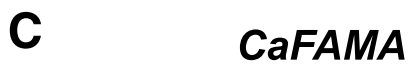

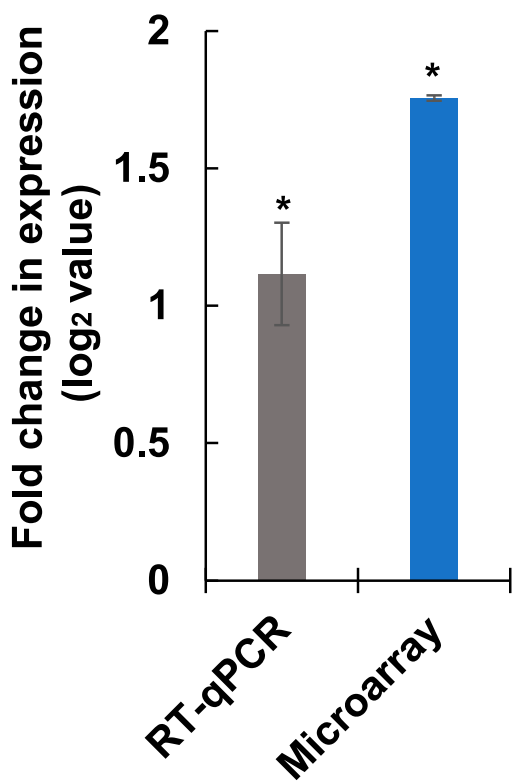

E

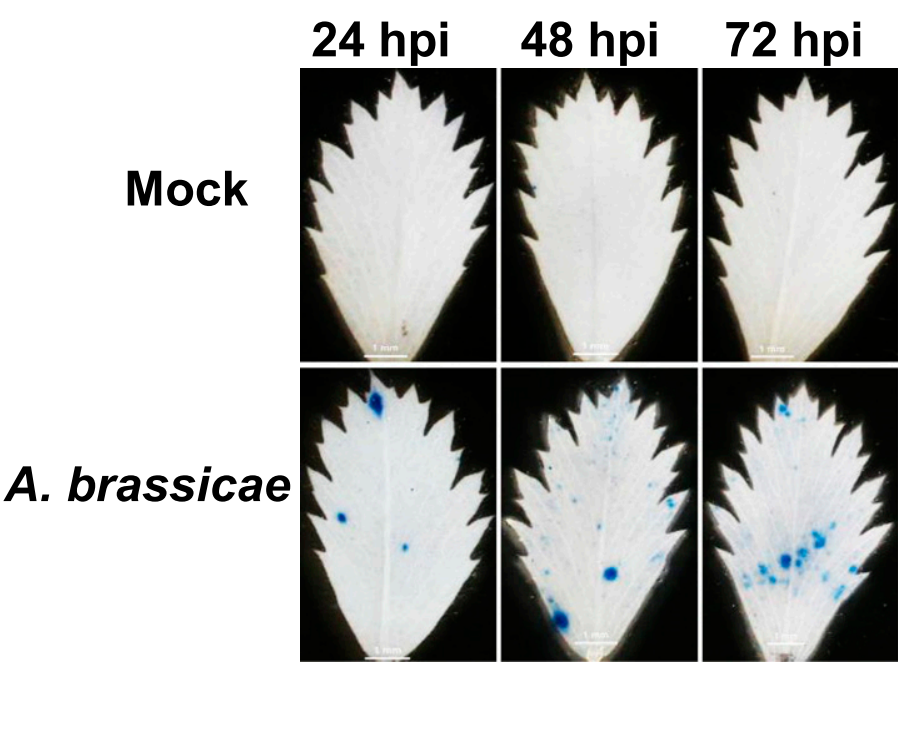

Fig. 7. Nonhost resistance in Cicer arietinum exhibits stomatal closure and cell death-mediated defense response against Alternaria brassicae. A to C, Transcript expression of the CaOST1 gene was quantified at $24 \mathrm{~h}$ postinoculation (hpi) and the expression of $C a P P 2 C A$ and $C a F A M A$ genes was quantified at $48 \mathrm{hpi}$ after inoculation with A. brassicae by microarray analysis and quantitative real-time PCR (RT-qPCR). For RT-qPCR, the expression values were normalized against the reference gene CaACTIN2. Graphs indicate the $\log _{2}$ fold change values calculated over the mock control. The data were obtained as the mean of three biological replicates. Error bars indicate the standard error of the mean. Student's $t$ test was applied and significant differences (at $P<0.05)$ were observed compared with the mock control (indicated by asterisks). D, The frequency of stomatal closure was observed by microscopic analysis in C. arietinum after inoculation with A. brassicae. The stomatal aperture index was calculated for mock-treated and A. brassicae-treated leaves at 24 and 48 hpi. Six leaves from three different plants were used for analyzing the stomata. One-way analysis of variance with Tukey's correction was applied; significant differences $(P<0.05)$ are indicated by different letters. E, Cell death was detected by trypan blue staining in $C$. arietinum plants inoculated with $A$. brassicae at 24,48 , and $72 \mathrm{hpi}$. The photographs were obtained using the 1× objective of a Stereozoom AZ100 microscope. Six biological replicates were used for estimating cell death. All the experiments were repeated twice, and consistent results were observed. 
of infection. The commonly upregulated genes at early and later timepoints included nucleo-binding site leucine-rich repeat (NBS-LRR) and RLK genes, which are involved in pathogen detection and downstream signaling in order to activate a robust defense response (De Young and Innes 2006; Greeff et al. 2012; Marshall et al. 2012; McHale et al. 2006). This suggests that the early detection of the pathogen and the timely activation of the downstream signaling cascade in $C$. arietinum could be the integral component involved in NHR mechanisms against $A$. brassicae. NHR mechanisms involve pre- or postinvasion layers of resistance against a pathogen (Gill et al. 2015; Heath 2000; Lee et al. 2017; Zellerhoff et al. 2010).

\section{Preinvasion NHR.}

In the preinvasion stage, the first step is conidial germination. Our histology study of A. brassicae showed that the process of conidiospore germination was delayed in $C$. arietinum compared with $B$. juncea. Consistent with earlier studies, the hyphal development and penetration events were well-established in the host plant one day after infection (Giri et al. 2013; Mandal et al. 2018; Tsuneda and Skoropad 1978). However, in C. arietinum, poor hyphal growth, suppressed appressorium formation, and limited penetration of hyphae were observed, indicating the involvement of preinvasion NHR in $C$. arietinum (Fig. 1). The preinvasion defense mechanisms preventing fungal penetration in nonhosts mainly involve processes like the accumulation of secondary metabolites, stomatal closure, cuticular wax deposition, and cell-wall thickening (Fonseca and Mysore 2019; Lee et al. 2017) (Fig. 8). Secondary metabolites, including phytoalexins, play a crucial role in inhibiting appressorium formation, hyphal development, and differentiation (Ahuja et al. 2012; Grayer and Harborne 1994; Iriti and Faoro

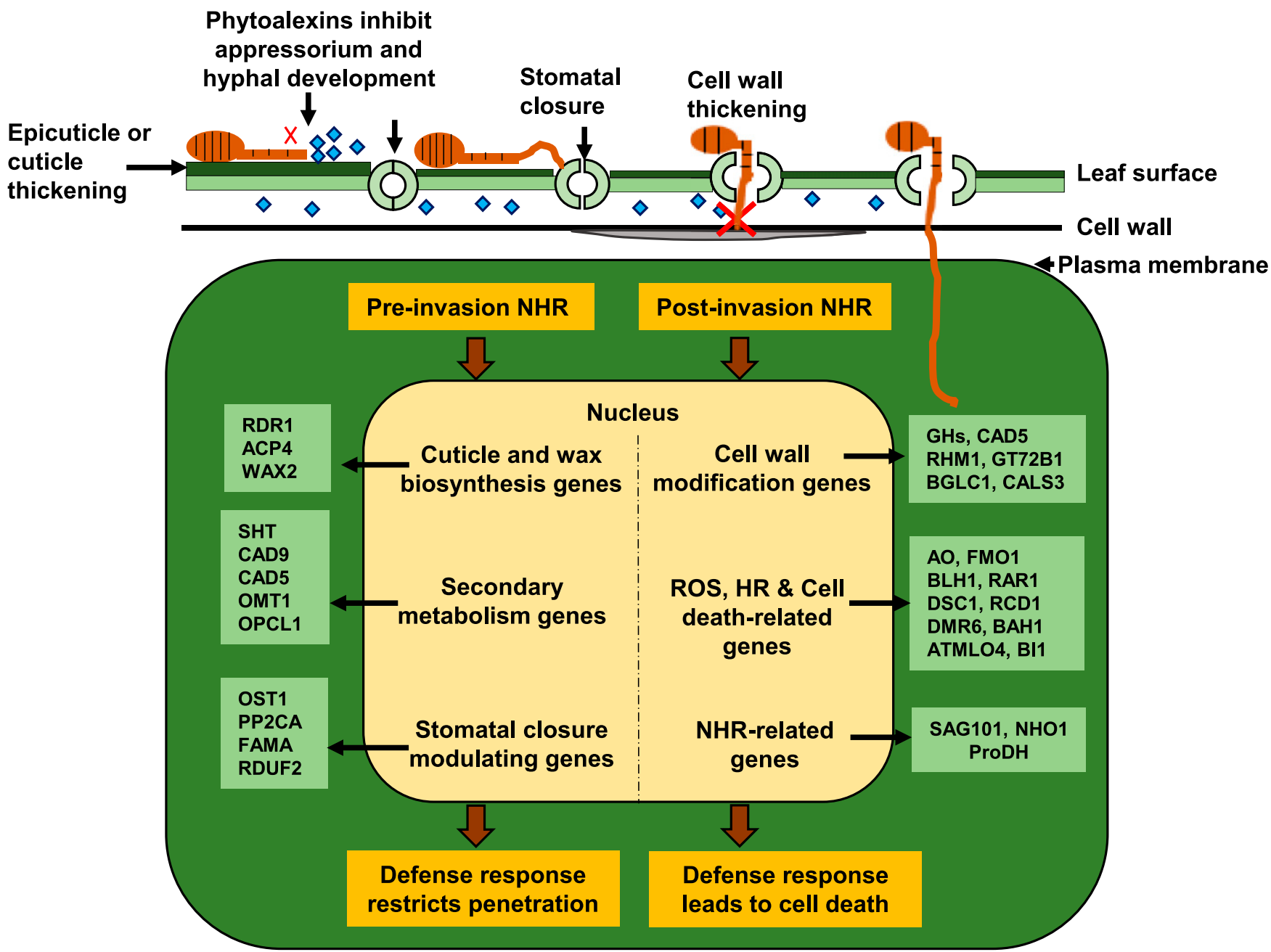

Fig. 8. A model of nonhost resistance of Cicer arietinum against Alternaria brassicae. The illustration depicts the genes involved in pre- or postinvasion nonhost resistance (NHR) in chickpea against A. brassicae. The genes with fold change $>1$ were identified and were categorized into the pre- or postinvasion layer of NHR based on the literature. During preinvasion NHR, the fungal pathogen fails to penetrate the plant. Genes encoding the proteins involved in stomatal closure, cuticle thickening by wax deposition, and secondary metabolite synthesis are highly induced after A. brassicae inoculation in C. arietinum. A. brassicae mainly enters through the stomata or, at some point, directly enters the cell after cuticle digestion. Our study indicated stomatal closure to be one of the crucial preinvasion NHR mechanisms that prevents pathogen entry into $C$. arietinum. Cuticle thickening may also contribute to limiting the direct penetration of the fungus in the plant. The accumulation of secondary metabolites such as phytoalexins on the leaf surface inhibits appressoria and hyphal development, which is required for fungal penetration. Furthermore, postinvasion NHR can halt fungal growth, even after the fungus enters the junctions between the epidermal cells. The cell wall acts as a barrier that prevents fungal entry inside the cell. The genes encoding proteins involved in cell-wall modification, including cell-wall thickening and lignification, are induced, indicating that chickpea prevents fungal entry inside the cell by reinforcing the cellwall modification machinery. We also found that postinvasion NHR in C. arietinum involves reactive oxygen species (ROS) accumulation and cell death to further prevent the growth of A. brassicae. Genes involved in ROS and cell death are induced in C. arietinum, leading to a robust defense response against A. brassicae. The genes encoding proteins involved in pre- and postinvasion resistance are shown in the model figure. Diamonds represent phytoalexins and indicate their secretion on the leaf surface and in the apoplastic space. 
2009; Ishiga et al. 2015; Mert-Türk 2002). Genes involved in secondary metabolism were upregulated in C. arietinum during early and late stages of pathogen infection. We speculate that poor hyphal growth and suppressed appressorium formation in $C$. arietinum might be due to the accumulation of secondary metabolites in the form of antimicrobial compounds. Furthermore, the stomata represent the primary entry point for A. brassicae penetration into the host plant (Giri et al. 2013; Mandal et al. 2018; Singh Saharan et al. 2016). Transcriptomic data from our study revealed that, in $C$. arietinum, the genes involved in stomatal closure, e.g., OST1 and PP2CA (Brock et al. 2010; Galbiati et al. 2011; Kim et al. 2018; Lim et al. 2015; Rusconi et al. 2013), were upregulated after inoculation with $A$. brassicae. PP2CA forms a complex with OST1 that contributes to ABA-mediated stomatal closure and its role has also been implicated in the plant defense response (Lim et al. 2015; Melotto et al. 2017; Toum et al. 2016). The role of ABA in stomatal immunity against pathogens has been well-studied (Lim et al. 2015). We observed the upregulation of ABAbiosynthesis genes after $A$. brassicae infection. During preinvasion NHR against $A$. brassicae, ABA might be involved in promoting stomatal closure through OST1 and $P P 2 C A$, which act as a core component of the ABA signaling pathway (Lim et al. 2015; Melotto et al. 2017). This was consistent with the microscopic observation of stomatal closure in $C$. arietinum upon inoculation with $A$. brassicae (Fig. 7B). Thus, the preinvasion NHR in $C$. arietinum involves stomatal closure to prevent the entry of $A$. brassicae (Fig. 8).

In addition, $A$. brassicae can sometimes directly enter the host plant by digesting the cuticle. However, only a few resistant host species with a high content of cuticular waxes can deter this (Conn and Tewari 1989; Skoropad and Tewari 1977). In $C$. arietinum, the genes involved in cuticular wax biosynthesis, RDRI and ACP4 (Leibman et al. 2018; Xia et al. 2009), were also upregulated after $A$. brassicae inoculation. Thus, the preinvasion NHR strategy of $C$. arietinum might also involve modulating the cuticular wax content on the plant surface to restrict A. brassicae entry. Cell-wall thickening and lignification restrict fungal entry inside the cell (Bellincampi et al. 2014; Lahlali et al. 2016). A. brassicae successfully penetrates cells by digesting the cell wall of the host plant (McRoberts and Lennard 1996). A tremendous upregulation of genes related to cell-wall modification was found following $A$. brassicae inoculation of $C$. arietinum, suggesting that the plant cell wall may act as a critical barrier against $A$. brassicae infection, leading to resistance. Our histopathological and transcriptome analysis findings collectively demonstrated the involvement of the preinvasion layer of NHR in C. arietinum against $A$. brassicae (Fig. 8).

\section{Postinvasion NHR.}

In $C$. arietinum, the frequency of hyphal penetration attempts was $10 \%$, indicating the presence of postinvasion NHR, which further arrests the growth of A. brassica inside the plant. ROS and defense-induced cell death are the critical components of postinvasion NHR (Fonseca and Mysore 2019; Ishiga et al. 2005; Lee et al. 2017). In the interaction of a nonhost plant and a necrotrophic fungal pathogen, ROS-mediated cell death is considered a crucial NHR mechanism (Azevedo et al. 2008; Ishiga et al. 2015; Loehrer et al. 2008). The NBS-LRR-based detection of pathogens leads to the downstream activation of PCD (Coll et al. 2011). Genes involved in ROS generation and cell death were upregulated in $C$. arietinum after $A$. brassicae inoculation. Consistent with this, DAB staining revealed the gradual accumulation of ROS in C. arietinum after A. brassicae inoculation. $\mathrm{H}_{2} \mathrm{O}_{2}$ accumulation has been found to initiate defense-induced cell death after pathogen infection in nonhost plants (Able et al. 2003; Hückelhoven et al. 2001; Lamb and Dixon 1997). Here, trypan blue staining showed localized cell death in C. arietinum at the infection site (Fig. 7C). The gradual increase in ROS accumulation is concurrent with the progression of localized cell death. Rapid defense-related cell death further limited growth and colonization of A. brassicae in C. arietinum. Thus, the postinvasion NHR mechanism operating in $C$. arietinum involves the accumulation of ROS accompanied by localized cell death (Fig. 8).

We surmise that the NHR mechanisms of $C$. arietinum against $A$. brassicae involve an aggressive preinvasion defense, followed by a moderate postinvasion defense. Several studies have reported the interspecific transfer of genes from nonhost to host plants to confer resistance against fungal pathogens (Lee et al. 2017). For example, interspecific transfer of the Arabidopsis WRR 4 gene encoding the toll interleukin 1 receptorNBS-LRR protein in B. napus and B. juncea confers disease resistance against the white rust pathogen Albugo candida (Borhan et al. 2010). In another study, the gene encoding stilbene synthase from grapevine was transferred into tobacco. Stilbene synthase is involved in the biosynthesis of resveratrol, a phytoalexin compound. The transgenic tobacco forming resveratrol confers durable resistance against Botrytis cinerea (Hain et al. 1993). Based on these reports, there is a possibility of utilizing some of the NHR-related genes identified in our study in developing Brassica transgenics carrying blightresistant genes. It would be interesting to test the functional relevance of these NHR-related genes, which can serve as promising candidates for developing blight-resistant Brassica cultivars.

\section{MATERIALS AND METHODS}

\section{Plant material and growth conditions.}

The Cicer arietinum cultivar PUSA372 and Brassica juncea (L.) Czern and Coss cultivar Varuna were used in this study. $C$. arietinum and $B$. juncea seeds were sown in pots $(5 \times 5$ inches in height and diameter) containing a $3: 1$ ( $\mathrm{vol} / \mathrm{vol}) \mathrm{mix}-$ ture of soil containing agro peat (Prakruthi Agri Cocopeat Industries, Bengaluru, India) and vermiculite (Keltech Energies Ltd., Nagpur, India). Pots were then transferred to a growth chamber (Conviron, Winnipeg, Canada) (8-h light and 12-h dark cycle) at $22^{\circ} \mathrm{C}$, with a constant photon flux light intensity of $200 \mu \mathrm{E} \mathrm{m}^{-2} \mathrm{~s}^{-1}$ and relative humidity of up to $70 \%$. The plants were irrigated alternately with water and Hoagland solution (Himedia Laboratories, Mumbai, India) thrice a week by pouring at the bottom of the pots, till the start of the stress treatment.

\section{Pathogen inoculum preparation and plant inoculation.}

A. brassicae had been isolated from infected leaves of the B. juncea cultivar Varuna grown in the field of the Indian Council of Agricultural Research Directorate of RapeseedMustard Research, Bharatpur, India. The details of this strain are also described by Swain et al. (2017). The fungus was maintained on the control host plant throughout the study. A pure single-spore culture was generated for each experiment on potato dextrose agar (PDA) (Himedia Laboratories). For inoculum preparation, A. brassicae was subcultured from 10-dayold culture on PDA medium incubated at $28^{\circ} \mathrm{C}$ in the dark (Supplementary Fig. S12). The conidial suspension was prepared in autoclaved distilled water by scraping the mycelia and spores from the surface of the actively proliferating fungal culture. The whole suspension was filtered, using a five-layered muslin cloth, to eliminate most of the mycelial debris. Next, the spore suspension was washed twice by centrifugation at $1,000 \times g$ for $10 \mathrm{~min}$ to obtain a clear suspension. The spores 
were resuspended in sterile water with $0.05 \%$ Tween-20 (Himedia Laboratories) as a wetting agent. By using a hemocytometer, the final concentration of the spore suspension was adjusted to $5 \times 10^{4}$ spores per milliliter for inoculation (Sharma et al. 2007). Leaves of 25-day-old $C$. arietinum and B. juncea plants $(n=10)$ were inoculated with the spore suspension by spraying until the whole plant was drenched entirely with the spore suspension. The inoculated plants were maintained at $22^{\circ} \mathrm{C}$ with $100 \%$ relative humidity. Control plants of $C$. arietinum and $B$. juncea were sprayed with sterile water containing the same amount of $0.05 \%$ Tween- 20 .

\section{Histopathological features of $\boldsymbol{A}$. brassicae infection.}

Infected $C$. arietinum and $B$. juncea leaf samples were collected at 12, 24, 48, and 72 hpi. The leaves were decolorized in acetic acid/ethanol (1:3) solution by overnight incubation at $25^{\circ} \mathrm{C}$. After this, the leaves were washed with autoclaved distilled water twice and. then. were stained with $0.05 \%$ lactophenol aniline blue for $30 \mathrm{~min}$. Lactophenol aniline blue comprised $20 \mathrm{ml}$ of phenol (Sigma Aldrich, St. Louis), $20 \mathrm{ml}$ of lactic acid (Fisher Scientific, Navi Mumbai, India), $40 \mathrm{ml}$ of glycerol (Fisher Scientific), $20 \mathrm{ml}$ of autoclaved distilled water, and $50 \mathrm{mg}$ of aniline blue (Himedia). After staining, the leaves were mounted on a glass slide with $40 \%$ glycerol. Observations were made by $100 \times$ bright field microscopy with a Nikon $80 \mathrm{i}$ microscope (Nikon Instruments Inc., Melville, NY, U.S.A.) fitted with a monochrome high-resolution digital camera. Quantitative observations, such as spore germination and hyphal length elongation, were made at $20 \times$ magnification and, for germ tube penetration and appressorium formation, at $40 \times$ magnification, using a Nikon $80 \mathrm{i}$ microscope. The photographs of disease symptoms of infected $C$. arietinum and B. juncea plants were taken at 7 dpi.

For Calcofluor staining, leaves were placed on a clean glass slide and one drop each of Calcofluor (Sigma Aldrich) and 10\% potassium hydroxide were placed over the leaf samples, followed by the mounting of cover slips. After $1 \mathrm{~min}$, the slides were examined, under a 4',6-diamidino-2-phenylindole filter at $380 \mathrm{~nm}$ excitation and $420 \mathrm{~nm}$ emission wavelengths, by a fluorescence microscope at $20 \times$ magnification (Nikon Instruments Inc.). For image analysis (for example, calculating the hyphal length), NIS Element software was used (Nikon).

\section{Transcriptome profiling by microarray analysis.}

Leaf tissues were harvested from mock control plants and pathogen-treated plants at 24 and 48 hpi. Microarray analysis for mock control and 24-hpi samples was performed in three biological replicates; two biological replicates were used for 48-hpi samples. One biological replicate was obtained by pooling leaf samples from four different plants, as suggested in a previous study (Kendziorski et al. 2005). RNA was isolated from the pooled leaf samples. The chip study was conducted at Genotypic Technology Pvt. Ltd. (Bangalore, India). A customized chickpea microarray chip (Agilent_AMADID037094; Agilent Technologies, Palo Alto, CA, U.S.A.) containing the 60-mer oligonucleotide probe was used. The source of the probe was RNA. The array specifications details for AMADID-37094 include, 62,976 as the total number of the probes, 1,319 as the total Agilent control probe. The total number of transcripts in chickpea were 43,389 (CTDB) (Verma et al. 2015), out of which 40,000 nonredundant transcripts were covered in the chip, corresponding to $92 \%$ of the transcriptome covered in the microarray study.

\section{RNA isolation.}

Total RNA for all the leaf samples was isolated using the TRIzol reagent (Invitrogen, Carlsbad, CA, U.S.A.), was purified using the RNeasy minikit (Qiagen, Hilden, Germany), and was quantified with a NanoDrop ND-1000 spectrophotometer (Thermo Scientific, Waltham, MA, U.S.A.). RNA quality was assessed using a Bioanalyzer (Agilent Technologies). Samples with RNA integrity numbers $>8$ were used in this study.

\section{Labeling.}

Double-stranded cDNA was prepared through reverse transcription of the total RNA (500 ng concentration). At $40^{\circ} \mathrm{C}$, the oligo dT primer tagged to a T7 polymerase promoter was used for cDNA synthesis. This cDNA was used as a template for cRNA synthesis. In vitro transcription was carried out, and the dye Cy3 CTP was inserted for cRNA synthesis. Labeling was performed with the Quick-Amp labeling kit (Agilent Technologies). cDNA synthesis and in vitro transcription were carried out at $40^{\circ} \mathrm{C}$. Qiagen RNeasy columns (Qiagen) were used for the purification of labeled cRNA. The quality and quantity were evaluated by Nanodrop ND-1000.

\section{Hybridization and scanning.}

At $60^{\circ} \mathrm{C}$, the labeled and purified cRNA (600 ng concentration) was fragmented. Then, the gene expression hybridization kit (Agilent Technologies) was used for the hybridization of cRNA on the chickpea GXP_8X60K microarray chip (AMADID: 037094). This step was done at $65^{\circ} \mathrm{C}$ for $16 \mathrm{~h}$ in a SureHyb microarray hybridization chamber (Agilent Technologies). Hybridized slides were washed with gene expression wash buffers (Agilent Technologies) and, then, were scanned using a microarray scanner (Agilent Technologies).

\section{Data analysis.}

The quantification of images was done by Feature extraction software (version 11.5; Agilent Technologies). Gene Spring GX software (version 12.1; Agilent Technologies) was used for the analysis of raw data. Microarray data normalization was performed using the RMA algorithm in Gene Spring GX, and differential expression values were obtained for each sample. Cluster analysis was performed after percentile shift normalization. Percentile shift normalization is a global normalization, in which the locations of all the spot intensities in an array are adjusted. This normalization considers each column in an experiment independently and computes the percentile of the expression values for this array, across all spots (where $n$ ranges from 0 to 100 and $n=75$ is the median). It subtracts this value from the expression value of each entity. Principal component analysis provides the correlation between the replicates of the microarray dataset for all samples. The FC expression values for pathogen-treated samples at 24 and 48 hpi were extracted with respect to the mock-treated samples as the control. For statistical analysis, the unpaired Student's $t$ test was used (Gene Spring GX version 12.1). The microarray data has been submitted to the National Center for Biotechnology Information (NCBI) Gene Expression Omnibus database (submission ID: GSE119865). For further analysis, the DEGs at both timepoints with FC $>1\left(\log _{2}\right)$ and $P<0.05$ were used for further analysis.

\section{Transcript annotation and expression analysis in silico.}

The annotation data for DEGs was obtained from the CTDB (Verma et al. 2015). A NCBI BLASTN search query was used for the annotation of a few other DEGs against the chickpea database (taxid: 3827). The hierarchical clustering of DEGs was performed using the Pearson coefficient correlation algorithm. The common and unique DEGs for both timepoints were viewed by generating Venn diagrams (Venny 2.1.0, Bioinfo GP, The National Center for Biotechnology Spanish National Research Council). For all the DEGs in chickpea, the Arabidopsis 
orthologous genes were obtained from CTDB (Verma et al. 2015). The TAIR GO tool was used for the classification of DEGs into different GO categories. More specific categorization of genes into different GO biological processes and GO enrichment analysis was performed by agriGO singular enrichment analysis, with the following TAIR10 settings in the background: statistical test method, Fisher; multi test adjustment method, false discovery rate with 0.05 level of significance (agriGO tool, functional pathway analysis was done by the MapMan Image Annotator module. FC values of DEGs were used for generating a heat map by using the Morpheus tool from GENE-E software.

\section{RT-qPCR analysis.}

RT-qPCR was performed, for the expression analysis of selected genes, by using the ABI Prism 7000 sequence detection system (Applied Biosystems, Foster City, CA, U.S.A.). Three biological replicates and two technical replicates were used. Total RNA was isolated from mock-treated and pathogen-treated leaf samples (100 to $150 \mathrm{mg}$ of fresh weight) by using TRIzol reagent (Thermo Fisher Scientific). The RNA quality and quantity were assessed by Nano Drop (Thermo Scientific). About $5 \mu \mathrm{g}$ of DNase-treated RNA was used for the synthesis of cDNA, using the Verso cDNA synthesis kit (ThermoFisher Scientific). The gene-specific primers were designed for RT-qPCR (Supplementary Table S9). The reaction mixture contained template cDNA, each of the specific primers (10 $\mathrm{mM}$ per milliliter), and SYBR green PCR master mix (Thermo Fisher Scientific). Cycle threshhold values obtained from CaActinl (EU529707.1), as the endogenous control, were used for data normalization (Reddy et al. 2016). FC values for all the genes were estimated by comparative cycle threshold $\left(\mathrm{C}_{\mathrm{T}}\right)$ values relative to those of the mocktreated samples (Livak and Schmittgen 2001). Student's $t$ test was used for evaluating the statistical significance at $P$ value $<0.05$.

\section{Measurement of stomatal aperture.}

Pathogen- and mock-treated leaf samples stained with lactophenol aniline blue (as described above) were used for examining the stomata. Stomata were observed at $40 \times$ objective under a bright field microscope (Nikon 80i). For image analysis and measurement, NIS Element software was used. The length and width of stomatal apertures were measured. The SAI was estimated by dividing the width of the stomatal aperture by the length of the stomatal aperture.

\section{Detection of ROS and cell death}

ROS detection was according to the protocol of ThordalChristensen et al. (1997), with slight modifications. Pathogenand mock-treated leaf samples were dipped in DAB solution $(1 \mathrm{mg} / \mathrm{ml})$ (Himedia) in $50 \mathrm{mM}$ Tris-acetate buffer at $\mathrm{pH} 5.0$, overnight in the dark at $25^{\circ} \mathrm{C}$. The leaves were then decolorized to remove chlorophyll in acetic acid/ethanol (1:3) solution overnight at $25^{\circ} \mathrm{C}$. Then, the leaves were mounted with $40 \%$ glycerol and were visualized at $1 \times$ magnification under a stereo-zoom microscope (Nikon AZ100; Olympus Corporation, Tokyo) mounted with a digital camera (Nikon Digital Sight DS-Ri1).

For cell death estimation, trypan blue staining was used. Pathogen- and mock-treated leaf samples were incubated, in $0.02 \%$ trypan blue solution (Himedia), overnight with constant shaking. After two washes with autoclaved distilled water, the leaves were destained in acetic acid/ethanol (1:3) solution overnight at $25^{\circ} \mathrm{C}$. The leaf samples were observed at $1 \times$ magnification under a stereo-zoom microscope (Nikon AZ100) mounted with a digital camera (Nikon Digital Sight DS-Ri1).

\section{Statistical analysis.}

The total number of biological replicates used in each experiment are given in legends of each figure. The error bars represent the values of mean \pm standard error of the mean. For statistical analysis, two-way or one-way analysis of variance with Tukey's correction was applied, and significant differences $(P<0.05)$ (indicated by different letters) were determined using SigmaPlot version 14.0 (Systat Software Inc., Chicago).

\section{ACKNOWLEDGMENTS}

We thank R. Tarafdar and S. Solanki for providing technical help at the laboratory and N. Singh, M. Patil, V. Murugan and A. Choudhary for critical reading of the manuscript. We acknowledge the Department of Biotechnology-eLibrary Consortium (DeLCON) and the National Institute of Plant Genome Research (NIPGR) library for providing access to e-resources, and the NIPGR Plant Growth Facility for plant growth support and space.

\section{AUTHOR-RECOMMENDED INTERNET RESOURCES}

agriGO website: http://bioinfo.cau.edu.cn/agriGO/analysis.php CABI Plantwise Knowledge Bank: www.plantwise.org/KnowledgeBank Chickpea Transcriptome Database (CTDB): http://www.nipgr.res.in/ctdb.html MapMan Image Annotator module: http://mapman.gabipd.org

Morpheus software: https://software.broadinstitute.org/morpheus

Nikon NIS-Element software:

https://www.microscope.healthcare.nikon.com/products/software/ nis-elements

The TAIR GO tool: https://www.arabidopsis.org/tools/bulk/go

\section{LITERATURE CITED}

Able, A. J., Sutherland, M. W., and Guest, D. I. 2003. Production of reactive oxygen species during non-specific elicitation, non-host resistance and field resistance expression in cultured tobacco cells. Funct. Plant Biol. 30:91-99

Ahuja, I., Kissen, R., and Bones, A. M. 2012. Phytoalexins in defense against pathogens. Trends Plant Sci. 17:73-90.

Azevedo, H., Lino-Neto, T., and Tavares, R. M. 2008. The necrotroph Botrytis cinerea induces a non-host type II resistance mechanism in Pinus pinaster suspension-cultured cells. Plant Cell Physiol. 49:386-395.

Bellincampi, D., Cervone, F., and Lionetti, V. 2014. Plant cell wall dynamics and wall-related susceptibility in plant-pathogen interactions. Front. Plant Sci. 5:228.

Bettgenhaeuser, J., Gilbert, B., Ayliffe, M., and Moscou, M. J. 2014 Nonhost resistance to rust pathogens - a continuation of continua. Front. Plant Sci. 5:664

Borhan, M. H., Holub, E. B., Kindrachuk, C., Omidi, M., BozorgmaneshFrad, G., and Rimmer, S. R. 2010. WRR4, a broad-spectrum TIR-NBLRR gene from Arabidopsis thaliana that confers white rust resistance in transgenic oilseed Brassica crops. Mol. Plant Pathol. 11:283-291.

Brock, A. K., Willmann, R., Kolb, D., Grefen, L., Lajunen, H. M., Bethke, G., Lee, J., Nürnberger, T., and Gust, A. A. 2010. The Arabidopsis mitogen-activated protein kinase phosphatase PP2C5 affects seed germination, stomatal aperture, and abscisic acid-inducible gene expression. Plant Physiol. 153:1098-1111.

Chen, L. Q. 2014. SWEET sugar transporters for phloem transport and pathogen nutrition. New Phytol. 201:1150-1155.

Chen, L. Q., Hou, B. H., Lalonde, S., Takanaga, H., Hartung, M. L., Qu, X. Q., Guo, W. J., Kim, J. G., Underwood, W., Chaudhuri, B., Chermak, D., Antony, G., White, F. F., Somerville, S. C., Mudgett, M. B., and Frommer, W. B. 2010. Sugar transporters for intercellular exchange and nutrition of pathogens. Nature 468:527-532.

Chen, L. Q., Qu, X. Q., Hou, B. H., Sosso, D., Osorio, S., Fernie, A. R., and Frommer, W. B. 2012. Sucrose efflux mediated by SWEET proteins as a key step for phloem transport. Science 335:207-211.

Cho, Y., Davis, J. W., Kim, K. H., Wang, J., Sun, Q. H., Cramer, R. A., Jr., and Lawrence, C. B. 2006. A high throughput targeted gene disruption method for Alternaria brassicicola functional genomics using linear minimal element (LME) constructs. Mol. Plant-Microbe Interact 19: 7-15.

Coll, N. S., Epple, P., and Dangl, J. L. 2011. Programmed cell death in the plant immune system. Cell Death Differ. 18:1247-1256. 
Conn, K. L., and Tewari, J. P. 1989. Interactions of Alternaria brassicae conidia with leaf epicuticular wax of canola. Mycol. Res. 93: 240-242.

Conn, K. L., Tewari, J. P., and Awasthi, R. P. 1990. A disease assessment key for Alternaria blackspot in rapeseed and mustard. Can. Plant Dis. Surv. 70:19-22

DeYoung, B. J., and Innes, R. W. 2006. Plant NBS-LRR proteins in pathogen sensing and host defense. Nat. Immunol. 7:1243.

Ellinger, D., and Voigt, C. A. 2014. Callose biosynthesis in Arabidopsis with a focus on pathogen response: What we have learned within the last decade. Ann. Bot. 114:1349-1358.

Fonseca, J. P., and Mysore, K. S. 2019. Genes involved in nonhost disease resistance as a key to engineer durable resistance in crops. Plant Sci. 279: 108-116.

Galbiati, M., Matus, J. T., Francia, P., Rusconi, F., Cañón, P., Medina, C., Conti, L., Cominelli, E., Tonelli, C., and Arce-Johnson, P. 2011. The grapevine guard cell-related VvMYB60 transcription factor is involved in the regulation of stomatal activity and is differentially expressed in response to ABA and osmotic stress. BMC Plant Biol. 11:142.

Gill, U. S., Lee, S., and Mysore, K. S. 2015. Host versus nonhost resistance: Distinct wars with similar arsenals. Phytopathology 105:580-587.

Giri, P., Taj, G., Meena, P. D., and Kumar, A. 2013. Microscopic study of Alternaria brassicae infection processes in Brassica juncea cultivars by drop plus agarose method. Afr. J. Microbiol. Res. 7:4284-4290.

Grayer, R. J., and Harborne, J. B. 1994. A survey of antifungal compounds from higher plants, 1982-1993. Phytochemistry 37:19-42.

Greeff, C., Roux, M., Mundy, J., and Petersen, M. 2012. Receptor-like kinase complexes in plant innate immunity. Front. Plant Sci. 3:209.

Greenberg, J. T., and Yao, N. 2004. The role and regulation of programmed cell death in plant-pathogen interactions. Cell. Microbiol. 6:201-211.

Hain, R., Reif, H. J., Krause, E., Langebartels, R., Kindl, H., Vornam, B., Wiese, W., Schmelzer, E., Schreier, P. H., Stöcker, R. H., and Stenzel, K. 1993. Disease resistance results from foreign phytoalexin expression in a novel plant. Nature 361:153-156.

Heath, M. C. 2000. Nonhost resistance and nonspecific plant defenses. Curr. Opin. Plant Biol. 3:315-319.

Hückelhoven, R., Dechert, C., and Kogel, K. H. 2001. Non-host resistance of barley is associated with a hydrogen peroxide burst at sites of attempted penetration by wheat powdery mildew fungus. Mol. Plant Pathol. 2:199-205.

Iriti, M., and Faoro, F. 2009. Chemical diversity and defence metabolism: How plants cope with pathogens and ozone pollution. Int. J. Mol. Sci. 10: 3371-3399.

Ishiga, Y., Takeuchi, K., Taguchi, F., Inagaki, Y., Toyoda, K., Shiraishi, T., and Ichinose, Y. 2005. Defense responses of Arabidopsis thaliana inoculated with Pseudomonas syringae pv. tabaci wild type and defective mutants for flagellin $(\Delta$ fliC) and flagellin glycosylation ( $\Delta$ orf1). J. Gen. Plant Pathol. 71:302-307.

Ishiga, Y., Uppalapati, S. R., Gill, U. S., Huhman, D., Tang, Y., and Mysore, K. S. 2015. Transcriptomic and metabolomic analyses identify a role for chlorophyll catabolism and phytoalexin during Medicago nonhost resistance against Asian soybean rust. Sci. Rep. 5:13061.

Jasalavich, C. A., Morales, V. M., Pelcher, L. E., and Seguin-Swartz, G. 1995. Comparison of nuclear ribosomal DNA sequences from Alternaria species pathogenic to crucifers. Mycol. Res. 99:604-614.

Kadian, A. K., and Saharan, G. S. 1983. Symptomatology, host range and assessment of yield losses due to Alternaria brassicae infection in rapeseed and mustard. Indian J. Mycol. Plant Pathol. 13:319-323.

Kendziorski, C., Irizarry, R. A., Chen, K. S., Haag, J. D., and Gould, M. N. 2005. On the utility of pooling biological samples in microarray experiments. Proc. Natl. Acad. Sci. U.S.A. 102:4252-4257.

Kim, T. W., Youn, J. H., Park, T. K., Kim, E. J., Park, C. H., Wang, Z. Y., Kim, S. K., and Kim, T. W. 2018. OST1 activation by the brassinosteroid-regulated kinase CDG1-LIKE1 in stomatal closure. Plant Cell 30:1848-1863.

Kolte, S. J. 1985. Diseases of annual edible oilseed crops. Vol. II, RapeseedMustard and Sesame Diseases. CRC Press Inc., Boca Raton, FL, U.S.A

Kolte, S. J. 2002. Diseases and their management in oilseed crops, new paradigm. Pages 244-252 in: Oilseeds and Oils-Research and Development Needs. M. Rai, H. Singh, and D. M. Hegde, eds. Indian Society of Oilseeds Research, Hyderabad, India.

Lahlali, R., Kumar, S., Wang, L., Forseille, L., Sylvain, N., Korbas, M., Muir, D., Swerhone, G., Lawrence, J. R., Fobert, P. R., Peng, G., and Karunakaran, C. 2016. Cell wall biomolecular composition plays a potential role in the host type II resistance to Fusarium head blight in wheat. Front. Microbiol. 7:910.

Lamb, C., and Dixon, R. A. 1997. The oxidative burst in plant disease resistance. Annu. Rev. Plant Physiol. Plant Mol. Biol. 48:251-275.
Lee, H. A., Lee, H. Y., Seo, E., Lee, J., Kim, S. B., Oh, S., Choi, E., Choi, E., Lee, S. E., and Choi, D. 2017. Current understandings of plant nonhost resistance. Mol. Plant-Microbe Interact 30:5-15.

Leibman, D., Kravchik, M., Wolf, D., Haviv, S., Weissberg, M., Ophir, R., Paris, H. S., Palukaitis, P., Ding, S. W., Gaba, V., and Gal-On, A. 2018 Differential expression of cucumber RNA-dependent RNA polymerase 1 genes during antiviral defence and resistance. Mol. Plant Pathol. 19:300-312.

Li, X., Yang, R., and Chen, H. 2018. The Arabidopsis thaliana Mediator subunit MED8 regulates plant immunity to Botrytis Cinerea through interacting with the basic helix-loop-helix (bHLH) transcription factor FAMA. PLoS One 13:e0193458.

Lim, C. W., Baek, W., Jung, J., Kim, J. H., and Lee, S. C. 2015. Function of ABA in stomatal defense against biotic and drought stresses. Int. J. Mol Sci. 16:15251-15270.

Livak, K. J., and Schmittgen, T. D. 2001. Analysis of relative gene expression data using real-time quantitative PCR and the $2^{-\Delta \Delta} \mathrm{C}(\mathrm{T})$ method. Methods 25:402-408.

Loehrer, M., Langenbach, C., Goellner, K., Conrath, U., and Schaffrath, U. 2008. Characterization of nonhost resistance of Arabidopsis to the Asian soybean rust. Mol. Plant-Microbe Interact 21:1421-1430.

Lu, M., Tang, X., and Zhou, J. M. 2001. Arabidopsis NHO1 is required for general resistance against Pseudomonas bacteria. Plant Cell 13:437-447.

Maeda, K., Houjyou, Y., Komatsu, T., Hori, H., Kodaira, T., and Ishikawa, A. 2010. Nonhost resistance to Magnaporthe oryzae in Arabidopsis thaliana. Plant Signal. Behav. 5:755-756.

Makandar, R., Nalam, V. J., Chowdhury, Z., Sarowar, S., Klossner, G., Lee, H., Burdan, D., Trick, H. N., Gobbato, E., Parker, J. E., and Shah, J. 2015 The combined action of ENHANCED DISEASE SUSCEPTIBILITY1, PHYTOALEXIN DEFICIENT4, and SENESCENCE-ASSOCIATED101 promotes salicylic acid-mediated defenses to limit Fusarium graminearum infection in Arabidopsis thaliana. Mol. Plant-Microbe Interact 28:943-953.

Mandal, S., Rajarammohan, S., and Kaur, J. 2018. Alternaria brassicae interactions with the model Brassicaceae member Arabidopsis thaliana closely resembles those with mustard (Brassica juncea). Physiol. Mol. Biol. Plants 24:51-59.

Marshall, A., Aalen, R. B., Audenaert, D., Beeckman, T., Broadley, M. R. Butenko, M. A., Caño-Delgado, A. I., de Vries, S., Dresselhaus, T., Felix, G., Graham, N. S., Foulkes, J., Granier, C., Greb, T., Grossniklaus, U., Hammond, J. P., Heidstra, R., Hodgman, C., Hothorn, M., Inzé, D., Ostergaard, L., Russinova, E., Simon, R., Skirycz, A., Stahl, Y., Zipfel, C., and De Smet, I. 2012. Tackling drought stress: Receptor-like kinases present new approaches. Plant Cell 24:2262-2278.

McHale, L., Tan, X., Koehl, P., and Michelmore, R. W. 2006. Plant NBSLRR proteins: Adaptable guards. Genome Biol. 7:212.

McRoberts, N., and Lennard, J. H. 1996. Pathogen behaviour and plant cell reactions in interactions between Alternaria species and leaves of host and nonhost plants. Plant Pathol. 45:742-752.

Meena, P. D., Awasthi, R. P., Chattopadhyay, C., Kolte, S. J., and Kumar, A. 2010. Alternaria blight: A chronic disease in rapeseed-mustard J. Oilseed Brassica 1:1-11.

Melotto, M., Zhang, L., Oblessuc, P. R., and He, S. Y. 2017. Stomatal defense a decade later. Plant Physiol. 174:561-571.

Mert-Türk, F. 2002. Phytoalexins: Defence or just a response to stress. J. Cell Mol. Biol. 1:1-6.

Miller, G., Suzuki, N., Ciftci-Yilmaz, S., and Mittler, R. 2010. Reactive oxygen species homeostasis and signalling during drought and salinity stresses. Plant Cell Environ. 33:453-467.

Mustilli, A. C., Merlot, S., Vavasseur, A., Fenzi, F., and Giraudat, J. 2002. Arabidopsis OST1 protein kinase mediates the regulation of stomatal aperture by abscisic acid and acts upstream of reactive oxygen species production. Plant Cell 14:3089-3099.

Mysore, K. S., and Ryu, C. M. 2004. Nonhost resistance: How much do we know? Trends Plant Sci. 9:97-104.

Nürnberger, T., and Lipka, V. 2005. Non-host resistance in plants: New insights into an old phenomenon. Mol. Plant Pathol. 6:335-345.

Oh, S. K., Lee, S., Chung, E., Park, J. M., Yu, S. H., Ryu, C. M., and Choi D. 2006. Insight into types I and II nonhost resistance using expression patterns of defense-related genes in tobacco. Planta 223:1101-1107.

Reddy, D. S., Bhatnagar-Mathur, P., Reddy, P. S., Sri Cindhuri, K., Sivaji Ganesh, A., and Sharma, K. K. 2016. Identification and validation of reference genes and their impact on normalized gene expression studies across cultivated and wild Cicer species. PLoS One 11:e0148451.

Rojas, C. M., Senthil-Kumar, M., Wang, K., Ryu, C. M., Kaundal, A., and Mysore, K. S. 2012. Glycolate oxidase modulates reactive oxygen species-mediated signal transduction during nonhost resistance in Nicotiana benthamiana and Arabidopsis. Plant Cell 24:336-352.

Rusconi, F., Simeoni, F., Francia, P., Cominelli, E., Conti, L., Riboni, M., Simoni, L., Martin, C. R., Tonelli, C., and Galbiati, M. 2013. The 
Arabidopsis thaliana MYB60 promoter provides a tool for the spatiotemporal control of gene expression in stomatal guard cells. J. Exp. Bot. 64:3361-3371.

Sharma, N., Rahman, M. H., Strelkov, S., Thiagarajah, M., Bansal, V. K., and Kav, N. N. 2007. Proteome-level changes in two Brassica napus lines exhibiting differential responses to the fungal pathogen Alternaria brassicae. Plant Sci. 172:95-110.

Shekhawat, K., Rathore, S. S., Premi, O. P., Kandpal, B. K., and Chauhan, J. S. 2012. Advances in agronomic management of Indian mustard (Brassica juncea (L.) Czernj. Cosson): An overview. Int. J. Agron. 2012: $1-14$.

Singh, R. K., Kumar, H., and Singh, A. K. 2010. Brassica based intercropping systems-A review. Agric. Rev. (Karnal) 3:253-266.

Singh Saharan, G., Mehta, N., and Meena, P. D. 2016. The disease. Pages 17-51 in: Alternaria diseases of crucifers: biology, ecology and disease management. Springer, Singapore.

Skoropad, W. P., and Tewari, J. P. 1977. Field evaluation of the epicuticular wax in rapeseed and mustard in resistance to Alternaria brassicae. Can. J. Plant Sci. 57:1001-1003.

Swain, D. M., Yadav, S. K., Tyagi, I., Kumar, R., Kumar, R., Ghosh, S., Das, J., and Jha, G. 2017. A prophage tail-like protein is deployed by Burkholderia bacteria to feed on fungi. Nat. Commun. 8:404.

Tewari, J. P. 1991. Structural and biochemical bases of black spot disease of crucifers. Adv. Struct. Biol. 1:325-349.

Thomma, B. P. H. J. 2003. Alternaria spp.: From general saprophyte to specific parasite. Mol. Plant Pathol. 4:225-236.

Thordal-Christensen, H., Zhang, Z., Wei, Y., and Collinge, D. B. 1997. Subcellular localization of $\mathrm{H} 2 \mathrm{O} 2$ in plants. $\mathrm{H} 2 \mathrm{O} 2$ accumulation in papillae and hypersensitive response during the barley-Powdery mildew interaction. Plant J. 11:1187-1194.

Toum, L., Torres, P. S., Gallego, S. M., Benavídes, M. P., Vojnov, A. A., and Gudesblat, G. E. 2016. Coronatine inhibits stomatal closure through guard cell-specific inhibition of NADPH oxidase-dependent ROS production. Front. Plant Sci. 7:1851.

Tronchet, M., Balagué, C., Kroj, T., Jouanin, L., and Roby, D. 2010. Cinnamyl alcohol dehydrogenases-C and D, key enzymes in lignin biosynthesis, play an essential role in disease resistance in Arabidopsis. Mol. Plant Pathol. 11:83-92.

Tsuneda, A., and Skoropad, W. P. 1978. Behavior of Alternaria brassicae and its mycoparasite Nectria inventa on intact and on excised leaves of rapeseed. Can. J. Bot. 56:1333-1340.

Verma, M., Kumar, V., Patel, R. K., Garg, R., and Jain, M. 2015. CTDB: An integrated chickpea transcriptome database for functional and applied genomics. PLoS One 10:e0136880.

Xia, Y., Gao, Q. M., Yu, K., Lapchyk, L., Navarre, D., Hildebrand, D., Kachroo, A., and Kachroo, P. 2009. An intact cuticle in distal tissues is essential for the induction of systemic acquired resistance in plants. Cell Host Microbe 5:151-165.

Yang, Z., Rogers, L. M., Song, Y., Guo, W., and Kolattukudy, P. E. 2005. Homoserine and asparagine are host signals that trigger in planta expression of a pathogenesis gene in Nectria haematococca. Proc. Natl. Acad. Sci. U.S.A. 102:4197-4202.

Zellerhoff, N., Himmelbach, A., Dong, W., Bieri, S., Schaffrath, U., and Schweizer, P. 2010. Nonhost resistance of barley to different fungal pathogens is associated with largely distinct, quantitative transcriptional responses. Plant Physiol. 152:2053-2066. 